This is an Open Access article, distributed under the terms of the Creative Commons Attribution licence (http://creativecommons.org/licenses/by/4.0/), which permits unrestricted re-use, distribution, and reproduction in any medium, provided the original work is properly cited.

\title{
Turbulence statistics in a negatively buoyant multiphase plume
}

\author{
Ankur D. Bordoloi ${ }^{1,5, \dagger}$, Chris C. K. Lai ${ }^{2}$, Laura Clark ${ }^{3}$, \\ Gerardo V. Carrillo ${ }^{4}$ and Evan Variano ${ }^{1}$ \\ ${ }^{1}$ Department of Civil and Environmental Engineering, University of California Berkeley, Berkeley, \\ CA 94720, USA \\ ${ }^{2}$ School of Civil and Environmental Engineering, Georgia Institute of Technology, Atlanta, \\ GA 30332, USA \\ ${ }^{3}$ Department of Civil and Environmental Engineering, Stanford University, Stanford, CA 94305, USA \\ ${ }^{4}$ Department of Civil and Environmental Engineering, Cornell University, Ithaca, NY 14853, USA \\ ${ }^{5}$ Institute of Earth Sciences, University of Lausanne, CH-1015, Lausanne, Switzerland
}

(Received 17 July 2019; revised 14 January 2020; accepted 18 April 2020)

We investigate the turbulence statistics in a multiphase plume made of heavy particles (particle Reynolds number at terminal velocity is 450). Using refractive-index-matched stereoscopic particle image velocimetry, we measure the locations of particles whose buoyancy drives the formation of a multiphase plume, together with the local velocity of the induced flow in the ambient salt-water. Measurements of the mean axial flow in the plume centreplane follow Gaussian profiles and that of the mean radial flow is consistent with integral plume theory. The turbulence characteristics resemble those measured in a bubble plume, including strong anisotropy in the normal Reynolds stresses. However, we observe structural differences between the two multiphase plumes. First, the skewness of the probability density function of the axial velocity fluctuations is not that which would be predicted by simply reversing the direction of a bubble plume. Second, in contrast to a bubble plume, the particle plume has a non-negligible fluid-shear production term in the turbulent kinetic energy (TKE) budget. Third, the radial decay of all measured terms in the TKE budget is slower than those in a bubble plume. Despite these dissimilarities, a bigger picture emerges that applies to both flows. The TKE production by particles (or bubbles) roughly balances the viscous dissipation, except near the plume centreline. The one-dimensional power spectra of the velocity fluctuations show a -3 power law that puts both the particle and bubble plume in a category different from single-phase shear-flow turbulence.

Key words: sediment transport, particle/fluid flows

\section{Introduction}

Plumes containing bubbles, particles and droplets are present in both environmental and industrial applications. A few examples of environmental interest are settling

$\dagger$ Email address for correspondence: aerials00@gmail.com 
sediment, volcanic eruption columns, $\mathrm{CO}_{2}$ ocean sequestration plumes, and rising oil droplets and gas bubbles from oil well blowouts (Freeth 1987; Baines \& Sparks 2005; Baines 2008; Socolofsky \& Bhaumik 2008; Woods 2010; Socolofsky, Adams \& Sherwood 2011; Huppert \& Neufeld 2014; Wang et al. 2016). Suspension-flow plumes differ from traditional single-fluid plumes in that the energy due to buoyant forcing is transmitted indirectly from the suspended phase to the continuous phase. The relative motion between the two phases introduces additional length and time scales; these must be included in the model formulation employed to predict plume behaviour, for example, when extending classic single-phase integral plume models (Morton, Taylor \& Turner 1956) to multiphase plumes (Milgram 1983; Sun \& Faeth 1986). Identifying such scales is non-trivial due to the complexity of particle-particle and particle-fluid interactions.

For the special case of air bubbles in water, empirical data collection has allowed accurate closure of predictive schemes (Lance \& Bataille 1991; Risso \& Ellingsen 2002; Mercado et al. 2010; Almeras et al. 2017; Risso 2018). However, the bubble-in-water plume can be quite different from other suspension-flow plumes of interest, because bubbles are far less dense than the ambient fluid (specific gravity of order $10^{-3}$ ) and have negligible inertia. Other plumes of interest are droplets in water (specific gravity of order 1), solid particles in water (specific gravity of order 2 to 10), or liquid droplets in air (specific gravity of order $10^{3}$ ). Each of these different suspensions can behave quite differently than the others in terms of the interphase interactions. Empirical data are relatively scarce for these other suspension-flow plumes.

Recent progress in suspension flows (especially turbulent flows) offers the hope that predictive techniques will eventually describe overall plume behaviour from a direct description of the internal dynamics of particle-fluid coupling. To help support this strategy and address some of the open questions that remain about turbulent suspension flows (Balachandar \& Eaton 2010; Guazzelli \& Morris 2012), we take an observational approach herein, measuring the particle and fluid behaviours in a particle-laden plume. Because particles are dynamically quite different from air bubbles in water, we are curious to see how the two-phase kinematics and the interstitial fluid turbulence behave.

Both bubbles and particles have been shown to modulate the turbulent properties of multiphase flows in a manner sensitive to volume fraction $(\phi)$, particle Reynolds number $\left(R e_{d}\right)$ and particle Stokes number $(S t)$. One starting point for understanding such turbulence modulation is the agitation of a quiescent fluid by bubbles or particles distributed homogeneously in space. This is often referred to as pseudo-turbulence (e.g. Lance \& Bataille (1991), Cartellier \& Rivière (2001)) as its characteristic scales are set by the bubble or particle wakes. Pseudo-turbulence is fundamentally anisotropic, with stronger velocity fluctuations aligned in the direction of suspended-phase motion ( $x_{1}$, typically vertical). Numerical simulations of pseudo-turbulence by Riboux, Legendre \& Risso (2013) show that the velocity fluctuation energy spectrum has a slope of $k^{-3}$ for wavelengths smaller than the integral length scale; they also find that the integral length scale is the ratio of the bubble or particle diameter and the drag coefficient, $C_{d}$.

A recent topical review article by Risso (2018) provides a comprehensive summary of pseudo-turbulence due to a homogeneous swarm of bubbles rising in a quiescent medium. First, the turbulence intensity is anisotropic and is dominated by the vertical component. The probability density function (p.d.f.) of the vertical component is positively skewed due to the wake immediately behind each rising bubble, whereas the 
other two components are symmetrically distributed with exponential tails. Because the turbulent kinetic energy (TKE) production in suspension flows occurs at two length scales (first at the scale of bubble wakes and second at the scale of overall bubble population), the combination of the two results in a $k^{-3}$ spectral subrange.

Previous laboratory (Hall et al. 2010) and numerical (Azimi, Zhu \& Rajaratnam 2011, 2012) studies have investigated the influence of particle size, particle concentration and nozzle size on the mean flow characteristics of sediment plumes. These authors considered fine to medium sized sand with diameters in the $0.1-0.8 \mathrm{~mm}$ range. Among all the observed differences between the particle plume and its single-phase counterpart, the nonlinear growth of plume width with downstream distances is a significant result; the two-phase plume initially spreads at approximately the same rate as single-phase plumes and then spreads at a lower rate beyond 60 nozzle diameters downstream. This change in the spreading rate affects all other mean flow variables as they are tied together by the continuity and momentum equations. In the present study, we consider the turbulent statistics of the two-phase plume in the initial spreading region. We choose to focus on the initial region because direct measurements of the plume turbulence are already lacking. Our results can be used for comparison in future studies looking at the region with a reduced plume growth rate.

To our knowledge, there are no existing studies examining turbulence in negatively buoyant particle plumes released into an unstratified, initially quiescent fluid. However, there are many studies of turbulence in bubble plumes with notable contributions coming from Soga \& Rehmann (2004), Wain \& Rehmann (2005), García \& García (2006), Seol, Duncan \& Socolofsky (2009), Simiano et al. (2009) and Lai \& Socolofsky (2019). These investigations reveal different velocity characteristics at a different axial distance $\left(x_{1}\right)$ from the origin. Simiano et al. (2009) examined the near-field characteristics $\left(x_{1} / D<2\right.$, where $D$ is the dynamic length scale to be defined in §3.1) and showed that the meandering nature of bubbles influences the effective spreading, volume fraction and mean velocity profiles. Seol et al. (2009) and Lai \& Socolofsky (2019) focused on the far field $\left(2<x_{1} / D<11\right)$ turbulence characteristics, such as Reynolds stress, turbulent transport and TKE budget; their results will be used to compare with our particle plume data.

In recent years, computational fluid dynamics (known as CFD) has seen significant improvements in computational speed, domain size and in the flow complexities it can simulate. This in turn has allowed more accurate representations of flow physics in the simulations using approaches such as large eddy simulation (LES) and hybrid Reynolds-averaged Navier-Stokes (known as RANS) LES. To further develop and improve turbulence closure models in these approaches, a comprehensive database of canonical turbulent flows is needed. This need is especially relevant for multiphase flows in which a physics-based coupling model between the carrier and dispersed phase is critical to successful predictions. To this end, we offer a dataset of a heterogeneous particle plume with statistics of the turbulent velocity fluctuations up to the third order. Although boundary layer resolving direct numerical simulations (DNS) that resolve all relevant flow physics have been available for several years now, these simulations tend to be limited to homogeneous flows with periodic boundary conditions. It is necessary to validate closure models using data with flow heterogeneities as most industrial and engineering flows are inhomogeneous. This study represents an important step as practical particle-laden flows are often released from a point source.

Characterizing the turbulence statistics in a multiphase plume is challenging, primarily due to the difficulty in simultaneously measuring velocity in both phases. 
Parameter

Length (mm)

Bulk volume $(\mathrm{ml})$

Density $\left(\mathrm{g} \mathrm{cm}^{-3}\right)$

Dynamic viscosity $\left(\mathrm{kg} \mathrm{m}^{-1} \mathrm{~s}^{-1}\right)$

Refractive index
Particle

(Teflon PFA)

2.0
110
2.1
-
1.34
Fluid

(0.4\% salt-water solution)

TABLE 1. Physical properties of particles and surrounding salt-water solution.

Traditional intrusive techniques such as hotwire anemometry suffer from the possibility of being damaged by the solid particles. Optical measurements from techniques such as particle image velocimetry (PIV) and laser Doppler anemometry are usually distorted due to the difference in optical properties of the two media. We overcome the distortion herein by carefully choosing two media with matched refractive indices.

This paper is organized as follows: $\S 2$ provides a detailed description of the plumegenerating facility and method for characterizing the two-phase flow. We report and discuss our experimental observations in $\S 3$. Finally, $\S 4$ provides the key conclusions from this work.

\section{Experimental set-up and methodology}

\subsection{Plume facility}

The plume experiments were conducted in a rectangular tank $(80 \mathrm{~cm}$ deep $\times 80 \mathrm{~cm}$ wide $\times 365 \mathrm{~cm}$ long) as described in Bordoloi \& Variano (2017). The tank was filled with tap water filtered to $5 \mu \mathrm{m}$ and maintained using an ultraviolet (UV) purifier. Then $91.1 \mathrm{~kg}$ of commercial sodium chloride (Cargill Top-Flo) was mixed to yield a salt concentration of $0.04 \mathrm{~g} \mathrm{ml}^{-1}$. The resulting solution has density $\rho_{s}=1.04 \mathrm{~g} \mathrm{~cm}^{-3}$ and kinematic viscosity $\mu_{s}=1.059 \times 10^{-3} \mathrm{~Pa}$ s (see table 1 ).

A schematic of the experimental set-up is shown in figure 1(a). The negatively buoyant plume was created by releasing $110 \mathrm{ml}$ of cylindrical Neoflon PFA AP-202 (a copolymer of tetrafluoroethylene and perfluoroalkyl vinyl ether) from a height of $56.5 \mathrm{~cm}$ via a screw feed particle release mechanism (see figure $1 \mathrm{~b}$ ). The pellets are right circular cylinders with length $=$ diameter $=2 \mathrm{~mm}$ (see figure $1 b$-A). The physical properties of the solid particles and the surrounding salt solution are summarized in table 1. Because of the hydrophobic nature of PFA, the particles tend to trap and hold air bubbles when added to water. To prevent these air bubbles from entering the experiment, particles were presoaked in water in a separate container and rapidly stirred to dislodge all bubbles. Once the particles were free of air bubbles, they were placed in a funnel for eventual release into the quiescent salt-water mixture. The funnel was kept partially submerged $23 \mathrm{~cm}$ below the free surface through a nozzle with internal diameter $d_{0}=11.25 \mathrm{~mm}$ so that the particles did not contact air (see figure $1 b$ ). Particle release was governed by a motor-driven helical screw. Prior to release, the particles were held inside the funnel by the blades of the screw. Upon release, the motor rotated the screw at a constant rate of 0.5 r.p.m. operated via Lego Mindstorms software. The particle flux, $Q_{0}=7.5 \mathrm{~cm}^{3} \mathrm{~s}^{-1}$ was measured by video recording the release of a known quantity of particles and measuring the time difference between the exit of the first and last particle from the funnel nozzle. Before each experiment, the tank fluid was seeded with optical tracers, specifically $13 \mu \mathrm{m}$ 
(a)

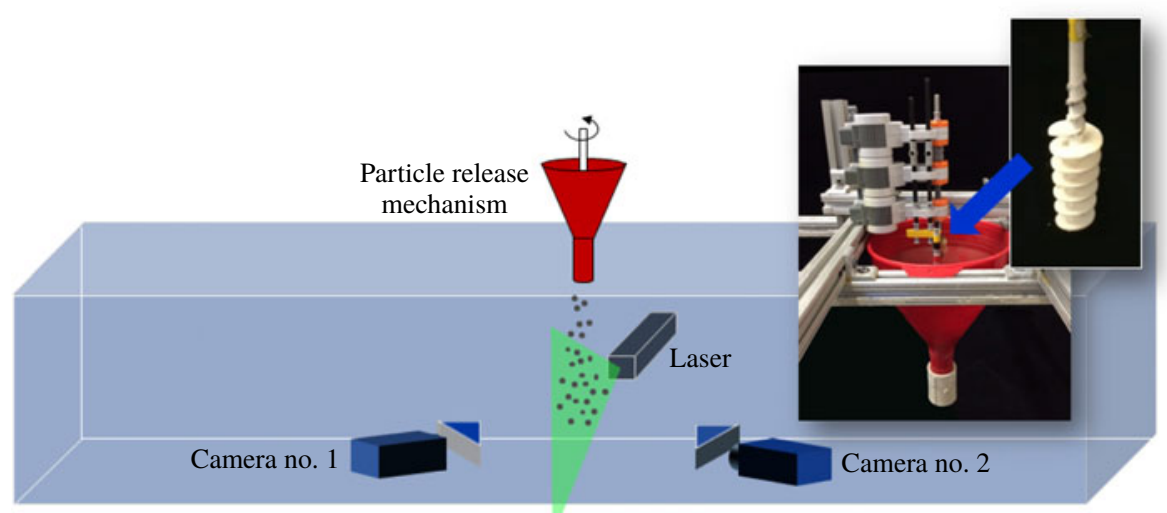

(b)

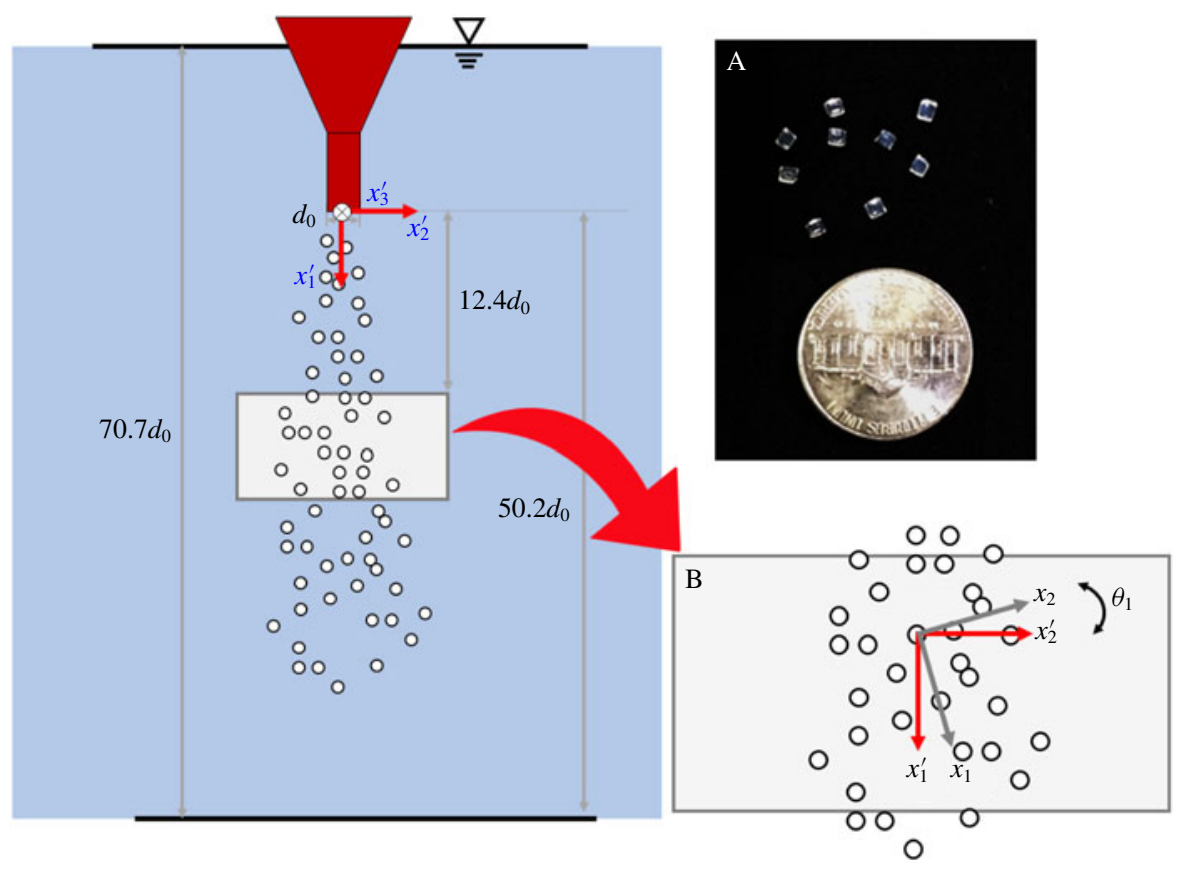

FIGURE 1. (a) Schematic of experimental facility and plume release mechanism (in inset), (b) regions of interest with specific dimensions, a picture of Teflon particles (inset A) and an illustration of two-dimensional transformation from world coordinates into the plume coordinates (inset B).

silver-coated hollow glass spheres (SH400S20, Conduct-O-Fil, Potters Industries). Twenty plume releases provided a sufficient number of samples for the analysis described in $\S 3$.

The plume release conditions for this study are parameterized into five nondimensional numbers as suggested in Lai et al. (2016) and are summarized in table 2. Here, $u_{0}$ is the initial plume velocity computed from particle volume flux $Q_{0}$ and $u_{s}$ is the characteristic settling velocity of a particle. We measure the inlet volume fraction $\left(\alpha_{0}\right)$ separately from a sample of $100 \mathrm{ml}$ of tightly packed particle-water mixture. The 


$\begin{array}{lcc}\text { Plume number } & N_{p}=\frac{u_{0}}{u_{s}} & \approx 0.2 \\ \text { Inlet volume fraction } & \alpha_{0} & \approx 0.65 \\ \text { Froude number } & F r=\frac{u_{0}}{\sqrt{g^{\prime} \alpha_{0} d_{0}}} & \approx 0.2 \\ \text { Plume Reynolds number } & \operatorname{Re}_{0}=\frac{u_{0} d_{0}}{v} & 550 \\ \text { Measurement distance } & x_{1} / d_{0} & 12.5-15\end{array}$

TABLE 2. Plume release parameters used in this study.

velocity scale $\left(u_{s}=0.23 \mathrm{~m} \mathrm{~s}^{-1}\right)$ is the terminal velocity of a single spherical particle in quiescent fluid and is computed using the standard Clift-Gauvin drag model for a sphere with volume equivalent to our particles. This value, inserted in the correction model proposed in Loth (2008), reduces significantly namely to $u_{s}=0.04 \mathrm{~m} \mathrm{~s}^{-1}$. Because computing the drag on a non-spherical particle in intermediate Reynolds number is complex, we restrict our analysis to the standardized drag model for a spherical particle. Future work in which we focus on particle velocities will examine this issue in greater detail.

\subsection{Refractive index matching}

To measure the fluid velocity inside and around the plume, we matched the refractive index $(n)$ of the particle and fluid phases. A target refractive index of PFA $(n \approx 1.34)$ was achieved from an aqueous solution of commercial sodium chloride with a concentration of $0.04 \mathrm{~g} \mathrm{ml}^{-1}$. The refractive index of the solution was measured to be 1.338 using an ATAGO refractometer and found to match with the salinity versus $n$ prediction given in Tan \& Huang (2015). Although the refractive indices of the two phases were matched, because of the scattering properties of PFA the particles appeared bright under the laser illumination. To limit the intensity of particles below the saturation threshold of the camera's sensor we used a circular polarizer on each camera lens. Figure 2 shows example images of Teflon particles in different salinity water samples illuminated by a laser sheet (wavelength $=532 \mathrm{~nm}$ ). The high-intensity elements in the images represent the particles intersected by the laser sheet. The low-intensity elements are the particles outside the laser-sheet plane. Fluid tracers (seen as tiny bright dots) are much more visible when particles and fluid have matched refractive indices (figure $2 b$ compared to figure $2 a$ ). This difference is well demonstrated inside the dotted rectangle in each figure: tracers obstructed by the out-of-plane particles are much more visible inside the rectangle in figure $2(b)$.

\subsection{Multiphase velocimetry}

We performed stereoscopic particle image velocimetry (SPIV) to find the twodimensional three-component (2D3C) velocity field of the fluid phase of the plume. The origin of our coordinate system is located at the tank centre. The plume is axisymmetric about the vertical axis, but we use Cartesian coordinates $\left(x_{1}, x_{2}, x_{3}\right)$ for our measurements as described in figure $1(b)$. Velocity measurement of the particle phase is currently underway and beyond the scope of this paper.

The $x_{3}=0$ plane was illuminated with a laser sheet $(1 \mathrm{~mm}$ thick at beam waist; Quantel/Big Sky Lasers, 532-nm double-pulse Nd-YAG). Two charge-coupled 
(a)

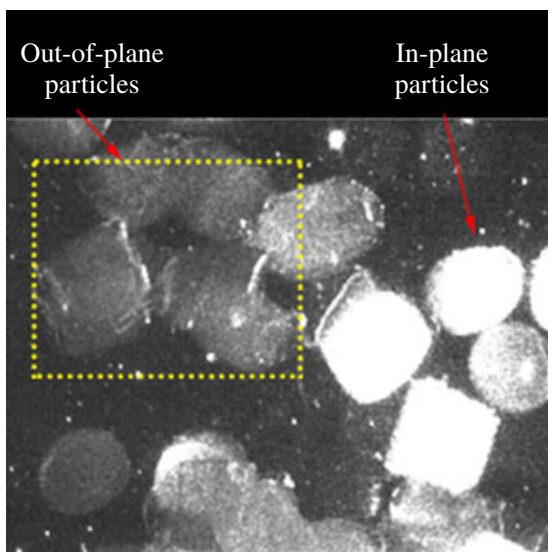

(b)

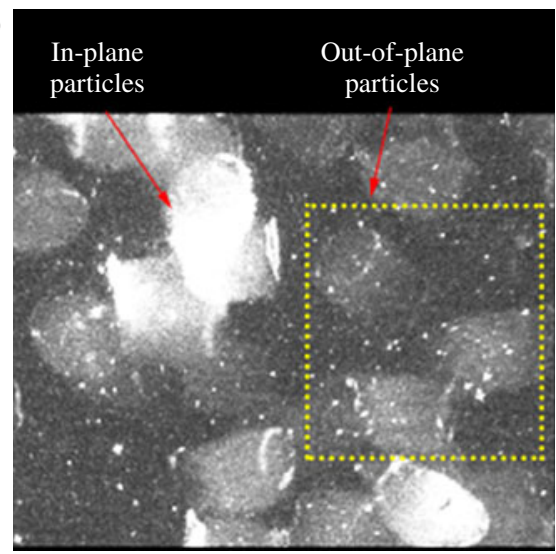

FIgURE 2. Teflon particles (large) and silver coated hollow glass spheres (small) illuminated by a $532 \mathrm{~nm}$ laser sheet in $(a)$ pure water, $(b) 0.4 \%$ salt-water mixture.

device (known as CCD) cameras (Imager PRO-X, 1600 pixels $\times 1200$ pixels) were synchronized with the laser pulses. They were placed $\pm 55^{\circ}$ to the laser sheet (cf. $90^{\circ}$ for standard 2D2C PIV). To minimize distortion due to this angle, water-filled acrylic prisms were placed between the camera lenses and the tank walls. The cameras were mounted with Nikkor $105 \mathrm{~mm}$ lenses, circular polarizers and Scheimpflug adapters. The interframe delay $(\Delta t)$ was optimized to $0.5 \mathrm{~ms}$. The PIV images were collected at a frequency of $14.0 \mathrm{~Hz}$. The sampling frequency was chosen such that the Eulerian fluid motions were decorrelated based on the correlation coefficient computed from subsequent samples. This set-up gave approximately 50 independent samples during the steady-state phase of each experimental run. We performed a convergence test for all dominant statistical quantities discussed in $\S 3$ and found them to converge within $\pm 4 \%$ for 1000 independent samples.

\subsubsection{Fluid-phase processing}

We computed the fluid-phase velocity field using DaVis 8.2 software (LaVision $\mathrm{GmbH}$ ). Before SPIV processing, we performed stereo self-calibration to reduce disparity in the alignment between the laser sheet and the measurement plane to below 0.1 pixel. Tracers and particles in an image were separated by intensity thresholding; erosion and dilation were applied to isolate individual particles. Fluid velocity fields were obtained by masking particles and correlating tracer locations through multipass stereoscopic cross-correlation. The cross-correlation was applied with an initial interrogation window of 64 pixel $\times 64$ pixel and a final window of 48 pixel $\times 48$ pixel with $50 \%$ overlap, yielding a spacing between vectors of $0.7 \mathrm{~mm}$. Interrogation windows were weighted according to the symmetric two-dimensional Gaussian function. Vectors were discarded based on universal outlier detection (Westerweel \& Scarano 2005) and left as data gaps. Figure 3 shows a representative field of fluid velocity fluctuations superposed with the corresponding particle image (transformed into the lab coordinate system). The grid cells are colour-coded with the out-of-plane velocity component while the vectors show the in-plane velocity field.

\subsubsection{Particle-phase processing}

To estimate the particle number density across the plume, we conducted a series of additional image processing steps (see figure 4). First, the raw images were 


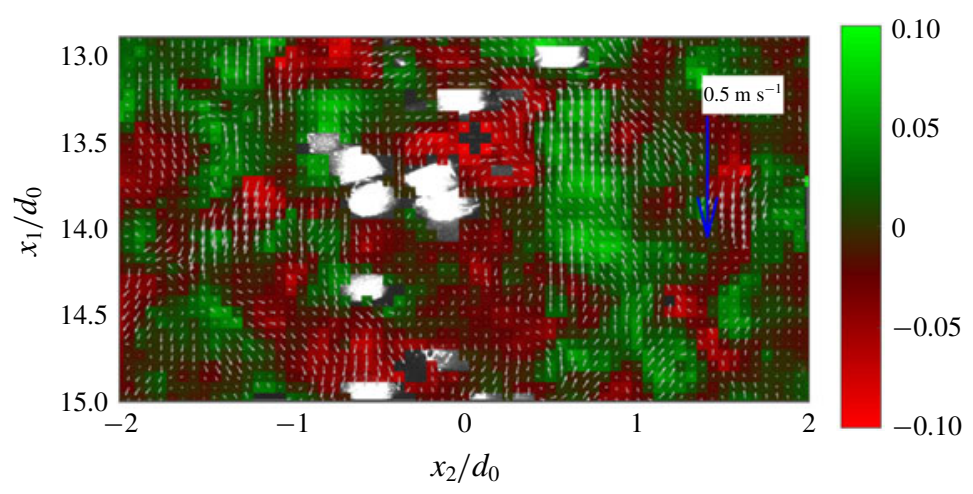

FIGURE 3. Sample mean-subtracted instantaneous 2D3C velocity field in the fluid phase superposed with the particle image in the laboratory coordinate system. The vectors show the in-plane velocity $\left(u_{1}, u_{2}, \mathrm{~m} \mathrm{~s}^{-1}\right)$, and the shades of red and green colours show the outof-plane velocity component $\left(u_{3}, \mathrm{~m} \mathrm{~s}^{-1}\right)$. The reference vector on the upper right corner corresponds to $0.5 \mathrm{~m} \mathrm{~s}^{-1}$.

transformed from the camera coordinate system to the lab coordinate system using the stereoscopic mapping function. Small-size background noise features (including tracers) were removed using a simple median filter.

In stereo mapping, after transforming into the lab coordinate system, the section of the particle intersected by the laser sheet should overlap in both cameras. Figure 4(a) shows a sample instant with the two camera images overlayed. The high intensity regions in the image represent the intersection between the two camera images, whereas the low intensity background is from the out-of-plane particles captured in only one of the two cameras. We remove the background noise by setting an intensity threshold and convolving the two images. The result is shown in figure $4(b)$.

After inspecting our dataset, we find many instances where particles are nearly touching as exemplified in the lower left pair in the image. The non-uniform intensity gradients in the bordering regions among different particle pairs yielded different intensity gradients. A traditional intensity-gradient-based image segmentation therefore could not differentiate between two particles, and so we adopted a segmentation technique based on the watershed transform. The watershed transform treats an image as a topographic map with brighter intensity pixels as heights, and finds the separating line that runs along the ridges (Gonzalez \& Woods 2007). The result, with individual particles identified with separate colours, is shown in figure $4(c)$. Since the area of a laser-intersected particle image is known a priori, we applied an area threshold to remove partially illuminated particles (which are out of the measurement plane). The centroids of particles identified from the final binary image are shown in figure $4(d)$.

\subsection{Coordinate transformation}

Typically in jets and plumes the cross-stream velocity components $\left(U_{2}\right.$ and $\left.U_{3}\right)$ are smaller than the axial velocity component $\left(U_{1}\right)$ near the centreline. Thus, any small misalignment between the plume axis and the measurement axis could lead to a systematic bias in the measured $U_{2}$ and $U_{3}$. The scenario is schematically shown in inset $\mathrm{B}$ in figure $1(b)$ in a simplified $x_{1}-x_{2}$ plane in which the plume axis, $x_{1}$, makes an angle $\theta_{1}$ with the measurement axis $x_{1}^{\prime}$. For our data, if $\theta_{1}$ is defined the same way 
(a)

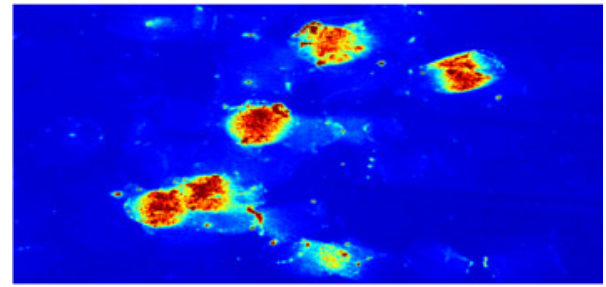

(c)

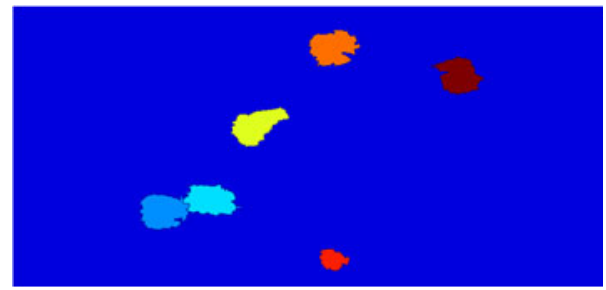

(b)

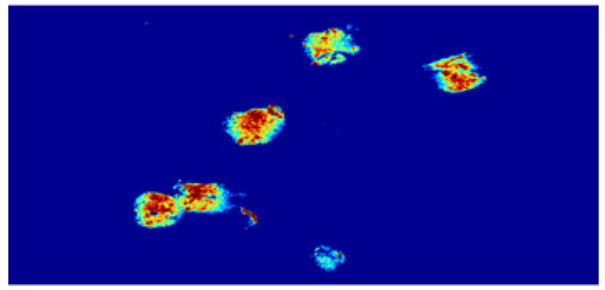

(d)

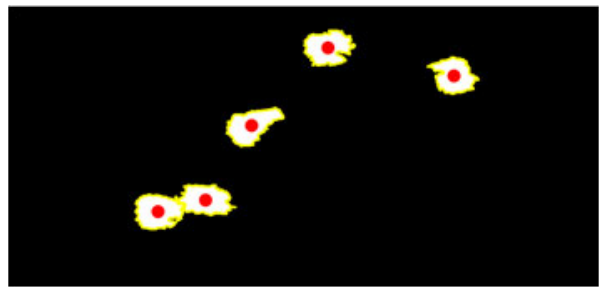

FIGURE 4. Image processing steps leading from raw PIV image to centroid identification: sample image showing $(a)$ particles from camera 1 and camera 2 blended into one image, (b) convolution of camera 1 image with camera 2 image, (c) image segmentation based on watershed transform and $(d)$ final binary image with particle boundaries and located centroids in the laboratory coordinate system.

and if $\theta_{2}$ is the angle between the plume axis and the measurement axis on the $x_{1}-x_{3}$ plane, using axisymmetry and assuming that $\left\langle U_{2}\right\rangle,\left\langle U_{3}\right\rangle=0$ at the plume centreline, the measured velocities can be transformed into the plume coordinate system via correction angles,

$$
\theta_{1}=\operatorname{atan}\left(\frac{\left\langle U_{2}^{\prime}\right\rangle_{0}}{\left\langle U_{1}^{\prime}\right\rangle_{0}}\right), \quad \theta_{2}=\operatorname{atan}\left(\frac{\left\langle U_{3}^{\prime}\right\rangle_{0}}{\left\langle U_{1}^{\prime}\right\rangle_{0}}\right)
$$

The measurement bias in $U_{2}$ and $U_{3}$ at the plume centreline are captured in figure $5(a)$ that shows the respective mean radial profiles, $\left\langle U_{2}^{\prime}\right\rangle$ and $\left\langle U_{3}^{\prime}\right\rangle$. We perform this transformation for the 20 replicate datasets independently with correction angles $\left(\theta_{1}, \theta_{2}\right)$ reported in figure $5(c)$. The bias in the non-zero centreline velocities are reflected in $\theta_{1}$ and $\theta_{2}$ which are small and less than $1.5^{\circ}$. The two velocity components transformed into the plume coordinate systems are shown in figure $5(b)$. The analysis that follows will be in the plume coordinate system.

\section{Results and discussion}

\subsection{Mean flow characteristics}

We first characterize the mean interstitial fluid velocity based on approximately 1000 independent PIV snapshots. The mean axial velocity field $\left\langle U_{1}\left(x_{1}, x_{2}\right)\right\rangle$ in dimensional form is shown in figure $6(a)$. The radial $\left(x_{2}\right)$ variations of $\left\langle U_{1}\right\rangle$ normalized by the local mean centreline velocity $\left\langle U_{c}\left(x_{1}, 0\right)\right\rangle$ (written as $U_{c}$ for simplicity from here onward) are shown in figure $6(b)$ at representative axial locations $\left(12.9 d_{0}<x_{1}<15.1 d_{0}\right)$ across our measurement region. The Gaussian plume radius, $b_{g}$, defined as the $x_{2}$ location where $\left\langle U_{1}\right\rangle=\mathrm{e}^{-1} U_{c}$, is obtained via a nonlinear least squares fit of each measured profile to a normalized Gaussian function. The data closely follow the Gaussian curve 

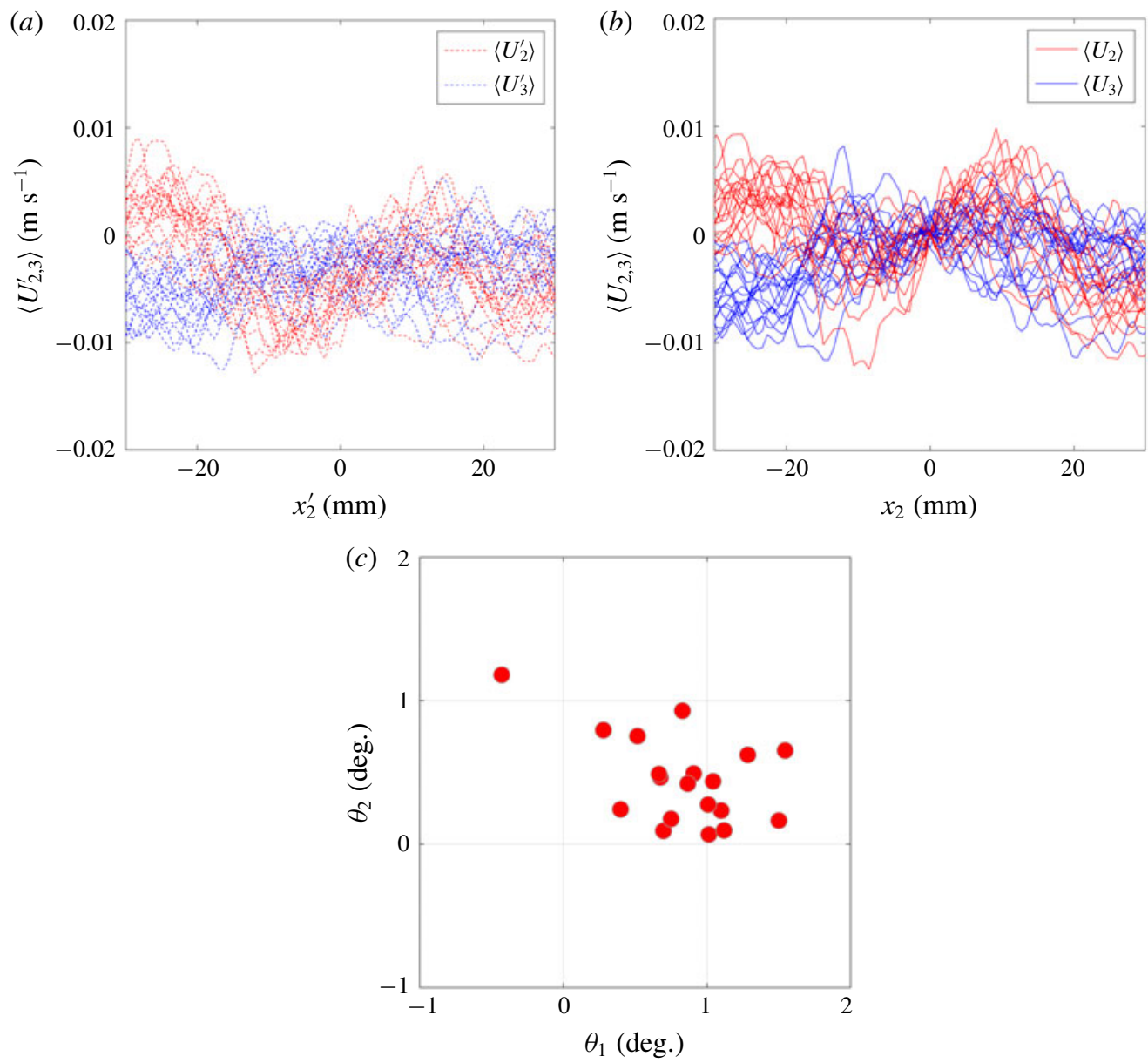

FIGURE 5. The radial and out-of-plane mean velocity components in $(a)$ laboratory coordinate and $(b)$ plume coordinate system averaged across the axial direction, and $(c)$ mapping angles $\left(\theta_{1}\right.$ and $\left.\theta_{2}\right)$ based on (2.1).

(solid line in figure $6 b$ ), which is also seen in single- and multi-phase jets and plumes (Milgram 1983; Darisse, Lemay \& Benaïssa 2012; Lai \& Socolofsky 2019).

Next, we examine the axial decay of centreline velocity, $U_{c}$, and the axial growth of Gaussian plume radius $\left(b_{g}\right)$ for a particle plume by comparing them to existing data from bubble plumes (see figure 7). For the purpose of comparison, we use two non-dimensional parameters: a velocity scale $\left(u_{s}\right)$ and a length scale $\left(D=g Q_{0} / 4 \pi \alpha_{0}^{2} u_{s}^{3}\right)$. These are based on an integral model of bubble plumes (Bombardelli et al. 2007), in which a constant $\alpha_{0}=0.083$ is used as the entrainment coefficient. The computed entrainment coefficient of our particle plume to be presented later is different from this a priori constant. The velocity scale $\left(u_{s}=0.23 \mathrm{~m} \mathrm{~s}^{-1}\right)$ is the terminal velocity of a single particle in quiescent fluid and is computed using a simple drag model (Clift, Grace \& Weber 1978). Based on $Q_{0}=7.5 \mathrm{~cm}^{3} \mathrm{~s}^{-1}$, the length scale $D$ is approximately $6.8 \mathrm{~cm}$. This situates our axial measurement region between $2-2.5 D$. 

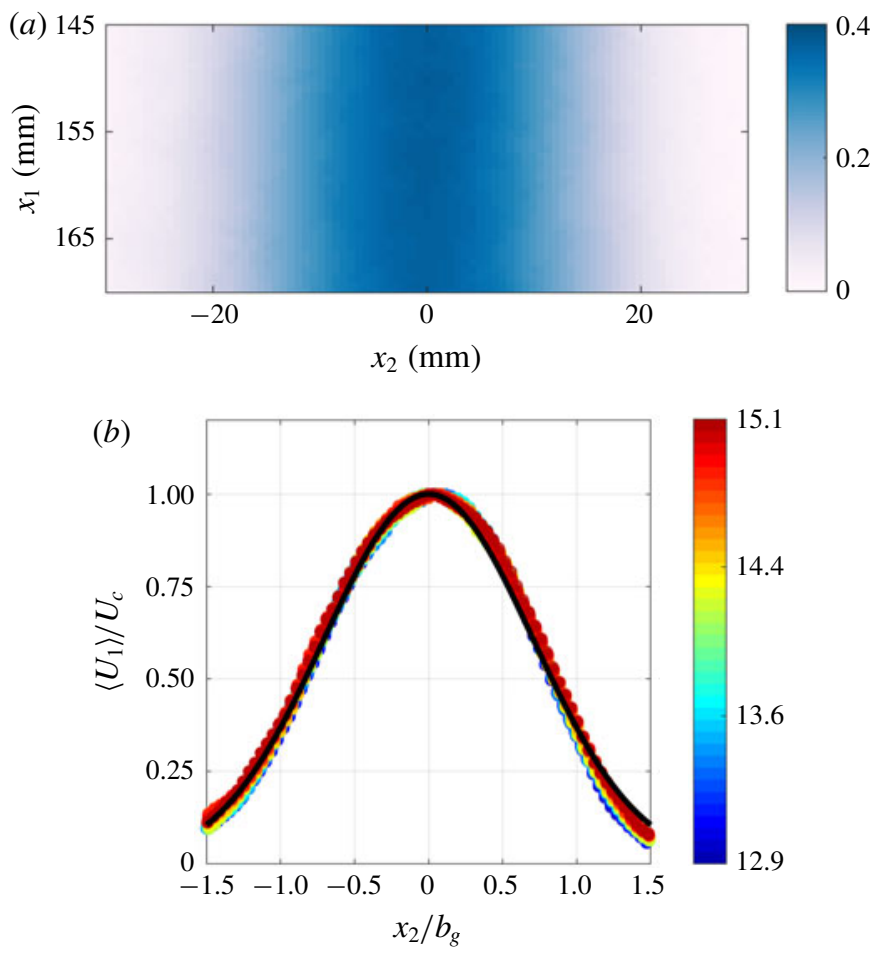

FIgURE 6. (a) Two-dimensional intensity field showing the local mean axial velocity $\left(\left\langle U_{1}\right\rangle, \mathrm{m} \mathrm{s}^{-1}\right)$ across the PIV measurement region, $(b)$ radial profiles of non-dimensional $\left\langle U_{1}\right\rangle / U_{c}$ at 45 axial locations $\left(x_{1} / d_{0}\right)$ indicated by the colourbar.

The overall trends in both centreline velocity and plume radius show similarity between particle and bubble plumes. The axial variation of $U_{c} / u_{s}$ in a particle plume is not measurable from our data, but we can say that it falls above the curve for bubble plumes (Lai \& Socolofsky 2019) (see figure 7a). The dashed and solid lines in figure $7(a)$ are the $-1 / 3$ power law fits, with $A=2.05$ (current data, see inset) and $A=1.6$ (Lai \& Socolofsky 2019). The variation of $b_{g}$ for all the data (including ours) is captured by the solid line $\left(b_{g} / D=0.114 x_{1} / D\right)$ in figure $7(b)$. Within the limited axial extent $(\approx 0.5 D)$ of our measurement, the local spreading rate $\left(\beta=\mathrm{d} b_{g} / \mathrm{d} x_{1}\right)$ is difficult to estimate. However, justified based on the collapse of our data on the solid line in figure $7(b)$, we will use $\beta=0.114$ in the remaining analyses in this paper.

Profiles of mean radial $\left(\left\langle U_{2}\right\rangle\right)$ and out-of-plane $\left(\left\langle U_{3}\right\rangle\right)$ velocities at various axial locations are shown in figures $8(a)$ and $8(b)$, respectively. The mean radial velocity exhibits typical jet/plume behaviour: within the plume radius $\left(\left|x_{2} / b_{g}\right|<1\right)$ the interstitial fluid flows away from the centreline, whereas outside the plume radius $\left(\left|x_{2} / b_{g}\right|>1\right)$ the surrounding fluid is entrained into the plume. The mean out-of-plane velocity should be zero by design (swirling motions are not introduced by the particle feeder) but it shows some variations and asymmetry far from the plume axis. The solid line in figure $8(a)$ is a nonlinear least squares fit to a shape function,

$$
\frac{\left\langle U_{2}\right\rangle}{U_{c}}=-\frac{\alpha}{\eta}\left(\left(1-\mathrm{e}^{-\eta^{2}}\right)-\frac{\beta}{\alpha} \eta^{2} \mathrm{e}^{-\eta^{2}}\right) .
$$



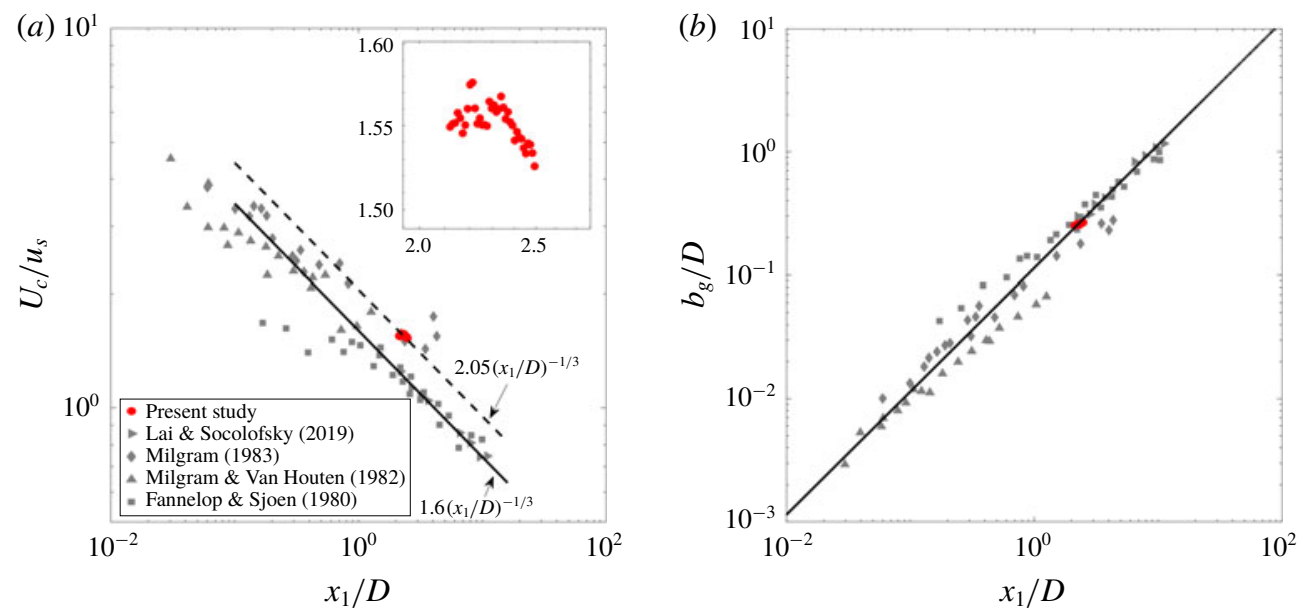

FIGURE 7. Comparison of $(a)$ mean centreline velocity, $U_{c}$, and (b) Gaussian plume radius, $b_{g}$, between a particle plume (current study) and bubble plume (published literature).
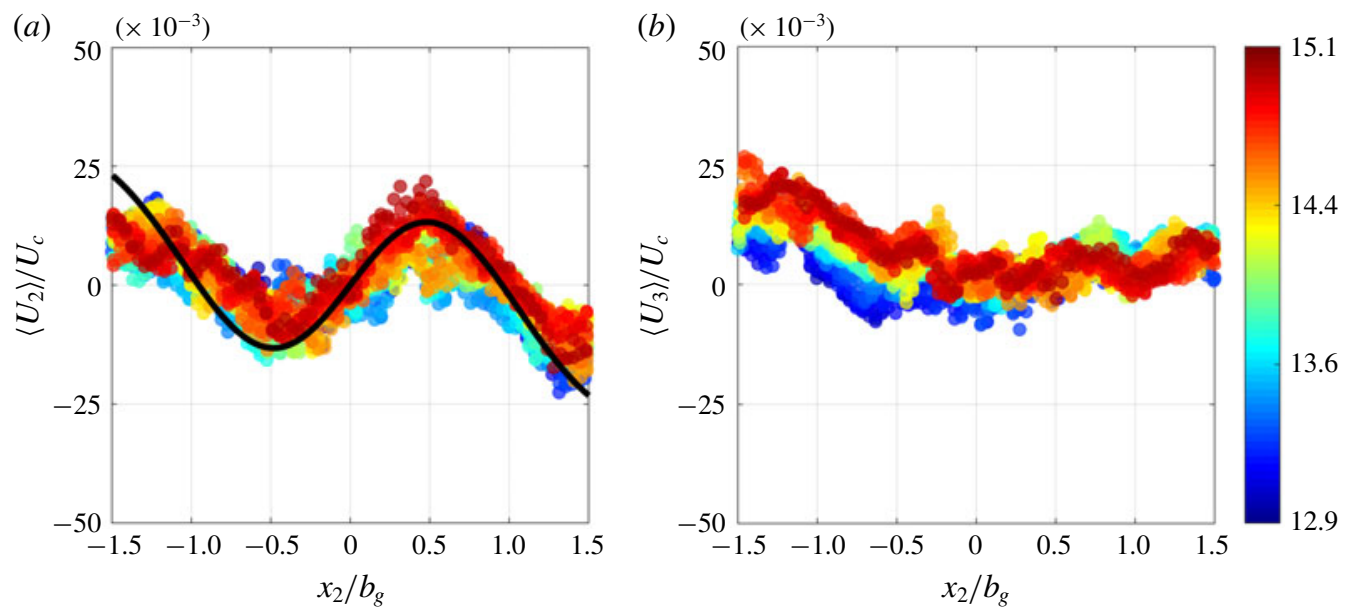

FIGURE 8. Radial profiles of non-dimensional $(a)\left\langle U_{2}\right\rangle / U_{c}$ and $(b)\left\langle U_{3}\right\rangle / U_{c}$ at various axial locations $\left(x_{1} / d_{0}\right)$ indicated by the colourbar.

Here, $\eta=x_{2} / b_{g}$. The shape function in (3.1) is obtained from the radial integration of the continuity equation written in a cylindrical coordinate system after adopting the entrainment hypothesis for jets/plumes and the Gaussian-profile assumption for $\left\langle U_{1}\right\rangle$ (Papanicolaou \& List 1988; Lee \& Chu 2003). Herein, the entrainment coefficient, $\alpha=0.07$ is computed as a fitting parameter in (3.1). The spreading versus entrainment ratio, $\beta / \alpha$, has been measured as 2 for pure jets (Lee $\&$ Chu 2003), and 1.2 for bubble plumes (Lai \& Socolofsky 2019). Our results show $\beta / \alpha=0.114 / 0.07=1.63$, situating the particle-laden plume between pure jets and bubble plumes.

We compute particle number density by counting the particles across each sample (detection method described in $\$ 2.3 .2$ ). The normalized distribution of particle number density $\phi$ across the radius of the plume is shown in figure 9. Here, $\langle\phi\rangle$ signifies 


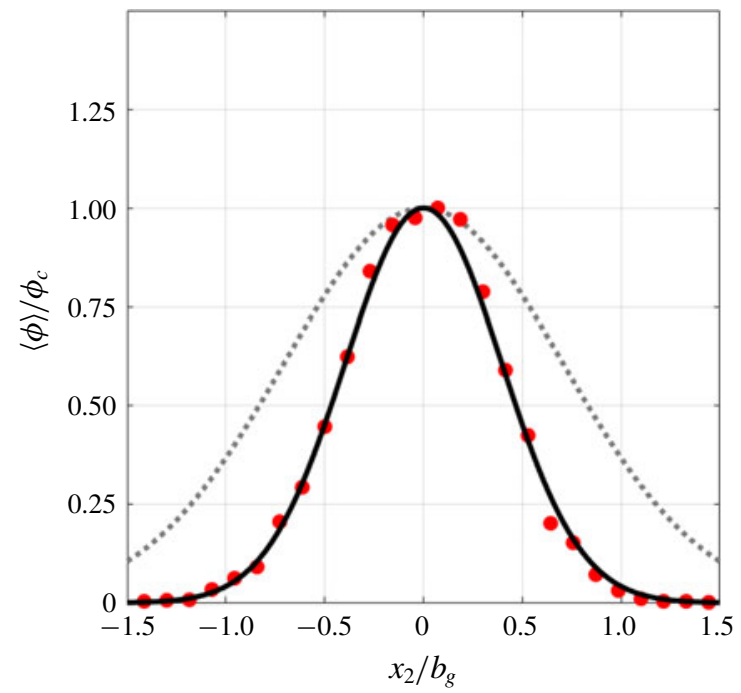

FIGURE 9. Normalized distribution of particle number density across the plume averaged along $x_{1}$. The solid line is a Gaussian fit to the measured data, and the dotted line is the Gaussian fit to mean axial fluid velocity $\left(\left\langle U_{1}\right\rangle / U_{c}\right)$ from figure 6 . The particle half-width, $b_{\phi}=0.56 b_{g}$.

the probability of finding a particle in the specified radial location, and $\phi_{c}$ is the centreline value, which measures as 0.11 at all axial locations. The distribution of $\phi$ allows us to fit a Gaussian profile and measure the half-width $\left(b_{\phi}\right)$, which we use to designate the 'particle core' from here onward. The axially averaged $b_{\phi}$ obtained from the particle concentration profiles, when normalized by the axially averaged $b_{g}$, shows that $b_{\phi} / b_{g}=0.56$. This ratio for the bubble plume in Lai \& Socolofsky (2019) was not measured, but in earlier studies of bubble plumes, e.g. Milgram (1983), reported values of $b_{\phi} / b_{g}$ are in the range of $0.8-0.9$. This value is somewhat higher than our value, implying that solid particles spread less rapidly than bubbles. This may be due to the fact that rising bubbles exhibit swirling motions and experience significant lateral lift force when compared to particles (Lai \& Socolofsky 2019). In addition, LES for a range of monodispersed and polydispersed bubble plumes in Fraga \& Stoesser (2016) show that the ratio $b_{\phi} / b_{g}$ is very sensitive to the size distribution of bubbles across the plume. They showed that the size distribution across the plume resembles a reverse-Gaussian profile with larger bubbles populating away from the centreline. This reveals the complexity related to clearly defining a bubble-core which depends on the distribution of the number density as well as bubble size across the plume. A future investigation on bubble plumes examining the relationship between polydispersity and turbulence characteristics will help to better explain the above differences between the particle and bubble plumes.

The dynamic length scale $D$ in the above formulation was derived by Bombardelli et al. (2007), wherein the authors started with the governing equations (continuity and momentum) of the air-water mixture, which were derived from a two-fluid approach (Buscaglia, Bombardelli \& Garcia 2002). The mixture was assumed to have low air void fractions, to be incompressible and the Boussinesq approximation for small density differences to be valid. For bubble plumes having air void fractions of the order of a few per cent, this is equivalent to the usual Boussinesq approximation 

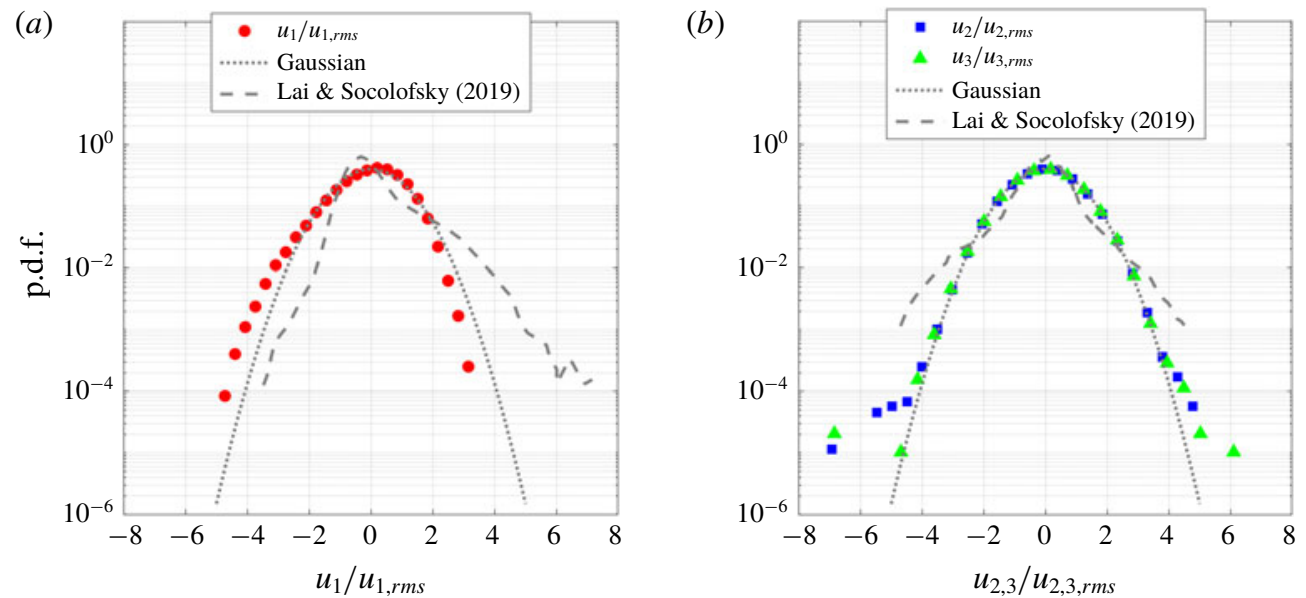

FIGURE 10. Comparison of standardized p.d.f. of $(a)$ axial, $u_{1} / u_{1, r m s} ;(b)$ radial, $u_{2} / u_{2, r m s}$, and out-of-plane, $u_{3} / u_{3, r m s}$, velocity fluctuations at the centreline of a particle plume (present data) and a bubble plume (Lai \& Socolofsky 2019). In (a), positive values indicate upward-moving fluid in the case of bubble plume and downward-moving fluid in the case of particle plume.

applied to single-phase water flows like seawater. The parameter $D$ was then obtained from the non-dimensional momentum equation by requiring a balance between inertia and buoyancy.

To apply the above formulation to particle-laden plumes, care must be taken to ensure that the Boussinesq approximation remains valid in the mean flow, and thus, imposing restrictions on the type of particles that can be considered. In our study, the specific gravity of Teflon particles relative to water is 2.15 . The mixture density equation gives a density difference $\phi\left(\rho_{s}-\rho_{w}\right)=\phi(2.15-1) \rho_{w}=1.15 \phi \rho_{w}$. The maximum solid fraction, $\phi$, occurs at the plume centreline and is approximately $10 \%$. This gives a density difference of approximately $10 \%,\left(\rho_{m}-\rho_{w}\right) / \rho_{w} \approx 0.1$. This $10 \%$ is a little bit on the high side but is not unreasonable. The possible mild violation of the Boussinesq approximation at the plume centreline should not invalidate the formulation that seeks to predict the overall, plume-scale dynamics.

\subsection{Fluctuating flow characteristics}

In this section, we examine the nature of the fluctuating components of velocity $\left(u_{i}=U_{i}-\left\langle U_{i}\right\rangle, u_{i, r m s}=\sqrt{\left\langle u_{i}^{2}\right\rangle}\right)$. The normalized p.d.f. of $u_{i}$ at the plume centreline is shown in figure 10. The respective p.d.f. for a bubble plume (Lai \& Socolofsky 2019) is also included in each plot for comparison. Interestingly, the distribution of the axial velocity fluctuations is negatively skewed for a particle plume, such that axial fluctuations opposite to the direction of particle motion are more common than those along the direction of particle motion. This behaviour is strikingly opposite to what is seen in bubble plumes and homogeneous bubble swarms (Riboux, Risso \& Legendre 2010; Prakash et al. 2016; Lai \& Socolofsky 2019), for which the axial velocity fluctuations moving in the direction of bubble motion are more common than those moving in the opposite direction. Another way to see this effect is that the mode of the distribution is slightly positive for the particle plume, while it is slightly negative for a bubble plume (see figure 10a). One implication of this contrasting 

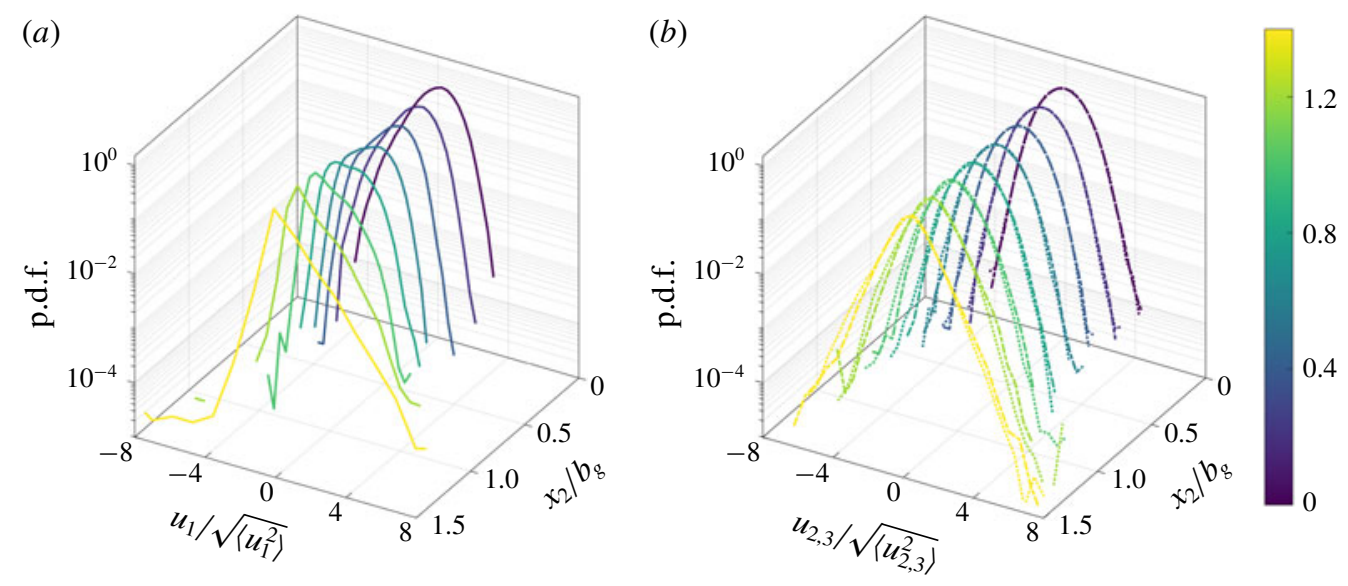

FIGURE 11. Standardized p.d.f. of $(a)$ axial, $u_{1} / u_{1, r m s} ;(b)$ radial, $u_{2} / u_{2, r m s}$; and out-ofplane, $u_{3} / u_{3, r m s}$ at various locations across a particle plume. The colourbar indicates the various radial locations $\left(x_{2} / b_{g}\right)$.

result is that the turbulence in a multiphase plume is sensitive to the direction of the plume with respect to gravity; particle plumes are not a simple reversal of bubble plumes. One implication of this contrasting result could be that the fluid flow in a multiphase plume is non-Boussinesq and is sensitive to the direction of the plume with respect to gravity. The cause of this difference could be the deformability of bubbles, the different density contrast between the two phases (2:1 for a particle plume and $0.001: 1$ for a bubble plume), or the different inertia between a bubble and a particle. All of these factors cause structural differences in the wake of a particle compared to that of a bubble and thus the role of role of added mass in these two flows. A future study comparing the wake-to-wake interaction in a particle plume with that in a bubble plume will help clarifying these differences between the two flows.

The p.d.f.s of radial and out-of-plane velocity fluctuations are symmetric about their means (figure 10b), and closely follow a Gaussian curve. We do not observe the prominent signatures of intermittency in the cross-stream components typically observed in bubble plumes and bubble swarms (Prakash et al. 2016; Lai \& Socolofsky 2019).

In figure 11, we further examine the velocity fluctuations across the plume at different radial locations $\left(x_{2} / b_{g}\right)$. For this purpose, we sort the data into eight equal bins (bin width $=0.16 b_{g}$ ) for $x_{2} \geqslant 0$ after checking that the choice of bin width does not change the results. Across the plume, the radial, $u_{2}$, and out-of-plane, $u_{3}$, velocity fluctuations are symmetric with nearly zero skewness (see figure $11 b$ ). The axial velocity fluctuations, $u_{1}$, switch from negatively skewed at the centreline (as discussed earlier) to positively skewed outside the half-radius $\left(x_{2}>0.5 b_{g}\right)$ of the plume. These characteristics are better captured in the statistical moments (r.m.s., skewness and kurtosis) as shown in figure 12. Figure 12(b) shows that the change of sign in $S\left(u_{1}\right)$ occurs at three-quarters of the plume width $b_{g}$, which also coincides with the maximum $u_{1, r m s}$ (see figure $12 a$ ). Both $u_{2, r m s}$ and $u_{3, r m s}$ show their maximum at the centreline, and are consistently smaller than $u_{1, r m s}$. The radial variation of kurtosis $K\left(u_{i}\right)$ in figure $12(c)$ captures the increasing flatness of each distribution outside the plume half-radius. 

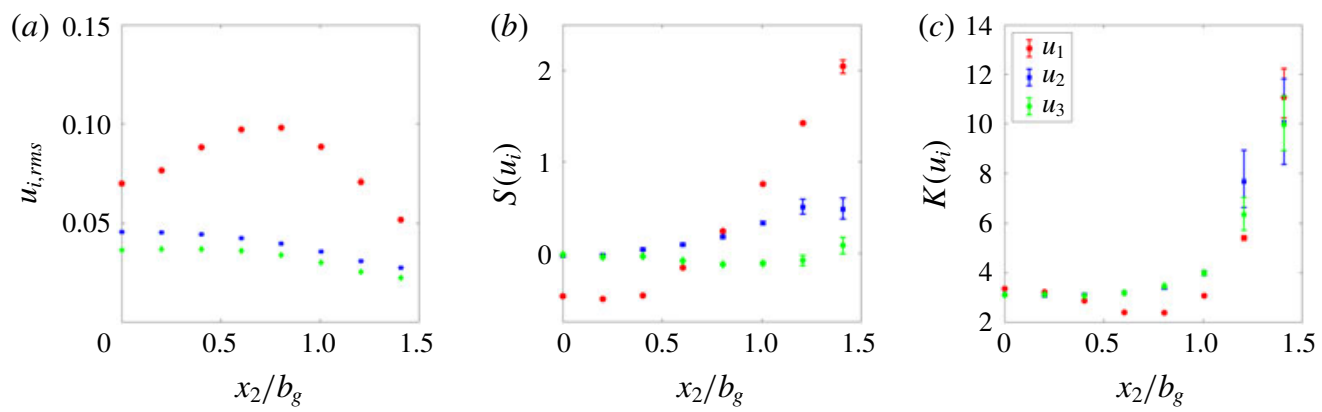

FIGURE 12. Higher-order statistics: (a) standard deviation; (b) skewness; and (c) kurtosis of the three components of velocity fluctuations across a particle plume.

\subsection{Reynolds stresses}

The Reynolds stresses across the plume are reported at all measured axial locations in figures 13(a) (normal stresses) and 13(b) (shear stress in the measurement plane). The turbulent kinetic energy $(k)$ based on 13(a) is shown separately in figure 13(c). A nonlinear least squares fit of the data to a shape-preserving function (see appendix A) captures well each profile (see fitted lines in figure 13). Results show that the turbulent kinetic energy is primarily dominated by the axial velocity fluctuations, and that it increases from the plume centreline to its maximum value near the edge of the plume $\left(x_{2} \approx 0.75 b_{g}\right)$. The maximum TKE located at $x_{2} / b_{g}=0.75$ is approximately $44 \%$ of $U_{c}^{2}$ and is approximately 1.5 times the TKE at the plume centre. The in-plane shear stress, $\left\langle u_{1} u_{2}\right\rangle / U_{c}^{2}$, follows a trend similar to that of a turbulent jet and increases from zero at the centreline to a maximum located near $x_{2} / b_{g}=0.75$ (see figure $13 b$ ). The location of maximum shear is consistent with that of $\left\langle u_{1}^{2}\right\rangle / U_{c}^{2}$, suggesting that the shear stress is dominated by the axial fluctuations $u_{1}$.

In figure 14, we compare the in-plane turbulent intensities normalized by the local mean axial velocity, $\left(\sqrt{\left\langle u_{1}^{2}\right\rangle} /\left\langle U_{1}\right\rangle\right)$, with two earlier studies on bubble plumes (Duncan, Seol \& Socolofsky 2009; Lai \& Socolofsky 2019). For this comparison, we use the shape functions (solid and dashed lines in figure 13(a)). The present data and the data from Duncan et al. (2009) show reasonably similar trends with unbounded growth away from the centreline as $\left\langle U_{1}\right\rangle$ approaches zero asymptotically outside the plume. The results from Lai \& Socolofsky (2019) show somewhat higher turbulent intensity inside the plume core. Also, the growth in their normalized turbulent intensities is not unbounded. Lai \& Socolofsky (2019) attribute this deviation to finite $\left\langle U_{1}\right\rangle$ outside of the plume caused by large recirculation cells caused by the tank walls; also shown in the LES simulations in Fraga \& Stoesser (2016). This created a non-zero upward mean flow and so the normalized stresses do not attain infinitely large values at the plume edges in their study. The centreline turbulent intensities for the present data are $18 \%$, $11 \%$ and $9 \%$ for $u_{1}, u_{2}$ and $u_{3}$, respectively. The axial turbulent intensity $\left(\sqrt{\left\langle u_{1}^{2}\right\rangle}\right)$ at the centreline for a bubble plume (Lai \& Socolofsky 2019) is also approximately twice the other two normal intensities $\left(\sqrt{\left\langle u_{2,3}^{2}\right\rangle}\right)$, suggesting stronger anisotropy in multiphase plumes when compared to a single-phase jets/plumes in which the ratio $\sqrt{\left\langle u_{1}^{2}\right\rangle /\left\langle u_{2,3}^{2}\right\rangle}$ is approximately 1.4 (Wang \& Law 2002). 

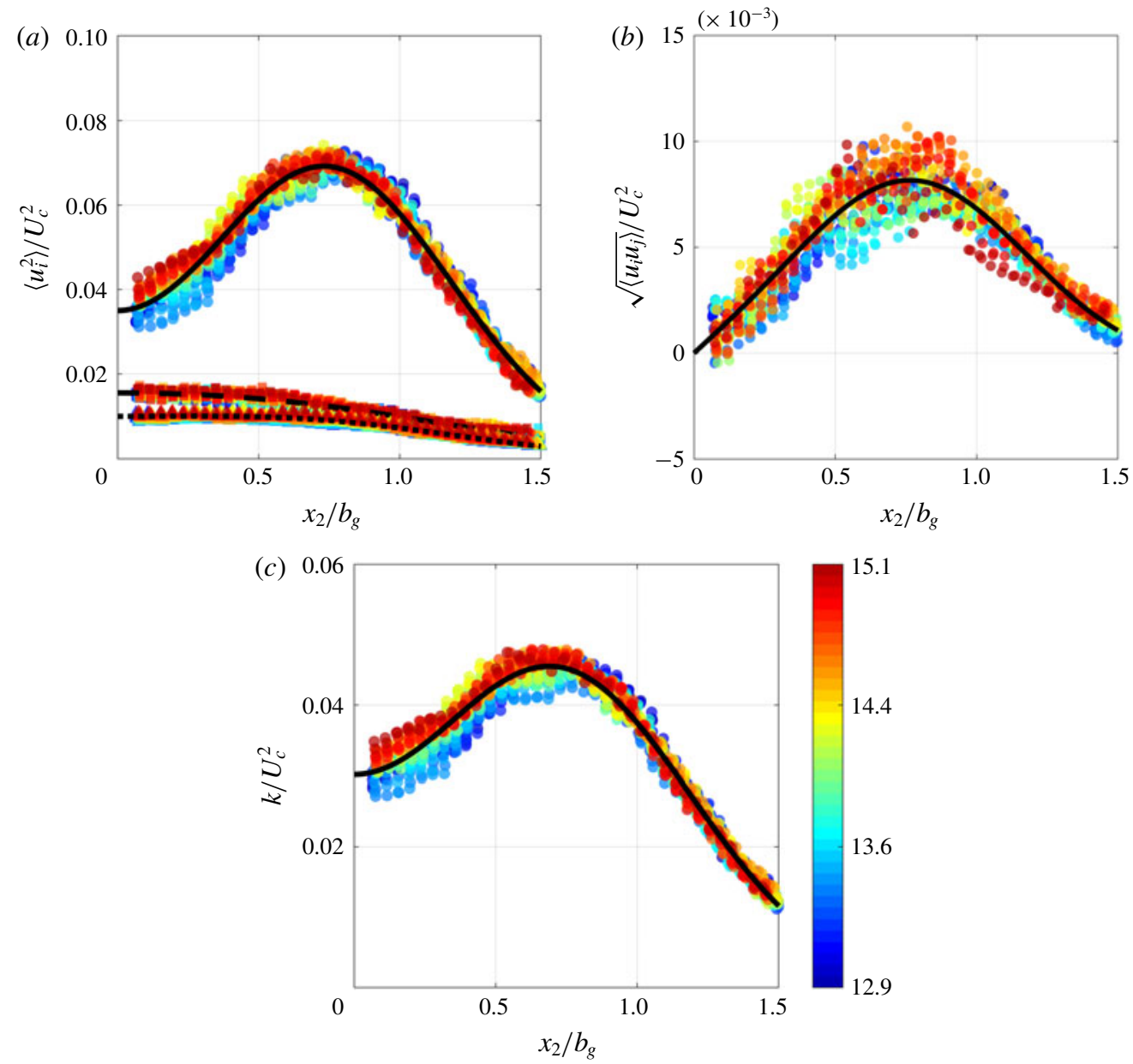

FIGURE 13. Radial profiles of normalized (a) turbulent normal stresses: $\left\langle u_{1}^{2}\right\rangle / U_{c}^{2},(\bigcirc)$; $\left\langle u_{2}^{2}\right\rangle / U_{c}^{2},(\square)$; and $\left\langle u_{3}^{2}\right\rangle / U_{c}^{2},(\triangle) ;(b)$ normalized turbulent shear stress $\left\langle u_{1} u_{2}\right\rangle / U_{c}^{2}$; and $(c)$ normalized turbulent kinetic energy $\left(k / U_{c}^{2}\right)$ at various axial locations. The colourbars show the normalized axial locations $\left(x_{1} / d_{0}\right)$ indicated by the colourbar.

\subsection{Conservation of kinematic momentum flux of the plume}

Using the method in Lai \& Socolofsky (2019), we assess the conservation of the total kinematic momentum flux, $M=\langle M\rangle+M_{t}$, of the induced liquid flow in our particle plume in figure 15 . Here, the momentum flux contributed by the mean flow is $\langle M\rangle=2 \pi \int_{0}^{\infty} x_{2} U_{1}^{2} \mathrm{~d} x_{2}$. The momentum flux carried by the turbulence fluctuations is computed as $M_{t}=2 \pi \int_{0}^{\infty} x_{2}\left(\left\langle u_{1}^{2}\right\rangle-0.5\left(\left\langle u_{2}^{2}\right\rangle+\left\langle u_{3}^{2}\right\rangle\right)\right) \mathrm{d} x_{2}$. The results are compared to the analytical expression for $M\left(x_{1}\right)$ for pure vertical plumes (Lee \& Chu 2003),

$$
M\left(x_{1}\right)=\left(3 \sqrt{2 \pi} \beta F_{0} / 4\right)^{2 / 3}\left(x_{1}-x_{0}\right)^{4 / 3} .
$$

Here, $x_{0}=-5.6 d_{0}$ is the virtual origin of the plume and $F_{0}=Q_{0} g$ is the buoyancy flux of the particles. Our results show reasonably good agreement with this model, suggesting that the particle plume obeys the scaling law $M \sim x_{1}^{4 / 3}$, which is consistent 

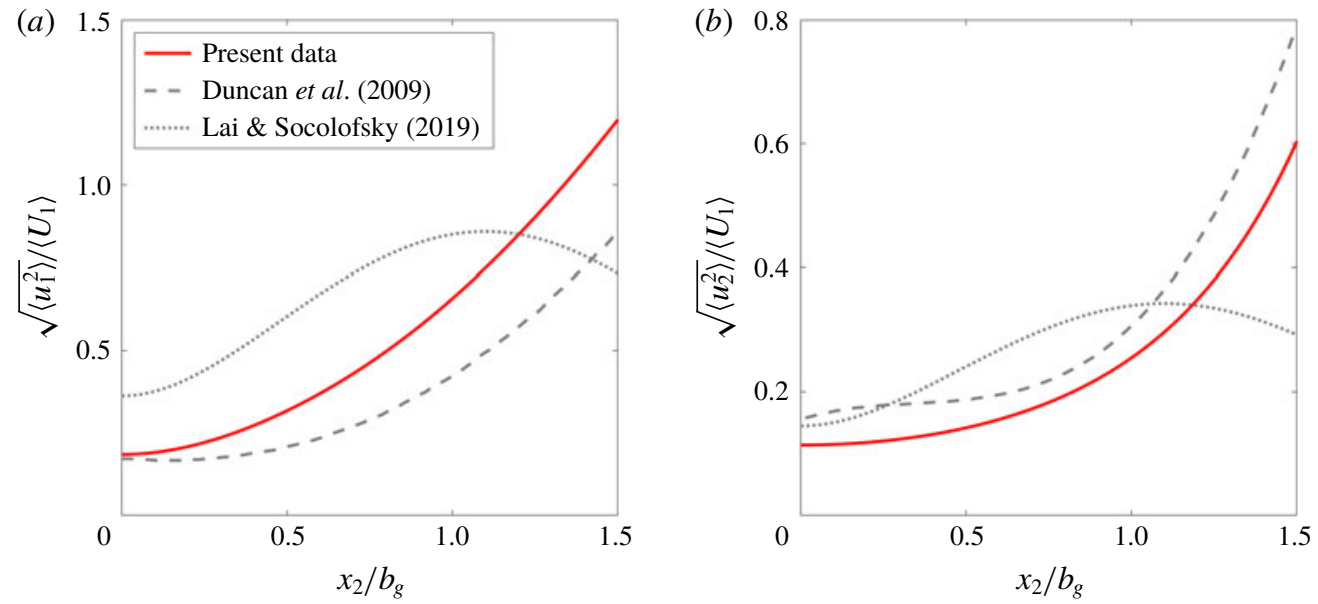

FIgURE 14. (a) Axial and (b) radial turbulence intensities normalized by the local mean axial velocity $\left\langle U_{1}\right\rangle$.

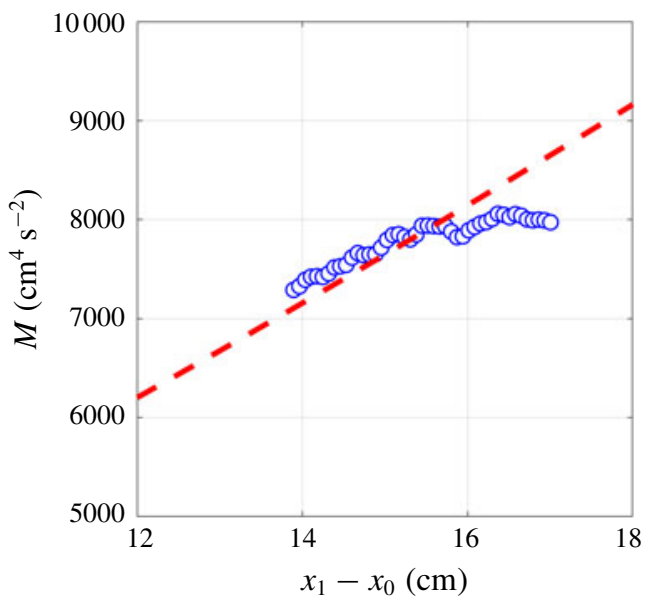

FIGURE 15. Variation of total kinematic momentum flux of the induced liquid flow in a particle plume along the axial direction. The dashed line shows a model prediction from (3.2).

with buoyancy-driven plumes. Some deviation from this power law is observed beyond axial location $x_{1}-x_{0}>16 \mathrm{~cm}$ where the value of $\langle M\rangle$ becomes almost flat. This is attributed to PIV measurement uncertainty near the edge of our measurement window. The total momentum flux is primarily contributed by the momentum flux due to the mean flow $\langle M\rangle$. The remaining contribution comes from $M_{t}$, and it is customary to quantify the contribution by the local momentum amplification factor $\gamma=M /\langle M\rangle$. For our particle plume, $\gamma$ is 1.2, averaged across all axial measurements. This result shows that $\gamma$ for a particle plume is larger than that for a single-phase jet/plume $(\gamma=1.07-1.09$ (Wang \& Law 2002)) and smaller than a bubble plume $(\gamma=1.4-1.6$ (Lai \& Socolofsky 2019)). 


$$
\begin{array}{ccc}
i & \left\langle\left(\frac{\partial u_{i}}{\partial x_{1}}\right)^{2}\right\rangle\left(\mathrm{s}^{-2}\right) & \left\langle\left(\frac{\partial u_{i}}{\partial x_{2}}\right)^{2}\right\rangle\left(\mathrm{s}^{-2}\right) \\
1 & 1555[1536,1577] & 1509[1487,1530] \\
2 & 624[619,629] & 565[561,568.5] \\
3 & 399[396,402] & 368.5[366,371]
\end{array}
$$

TABLE 3. The second moment of the six available components of the velocity gradient tensor averaged axially and radially. The quantities within the bracket indicate the $95 \%$ uncertainty bounds obtained with the bootstrap method.

\subsection{Velocity triple correlation and turbulent transport}

Because the p.d.f. of axial velocity fluctuations in a particle and a bubble plume are oppositely skewed (see figure 10a), some interesting differences between the two flows can be identified in terms of the velocity triple-correlation terms. These terms contribute to the transport of turbulent kinetic energy and thus are important in the TKE budget ( $\$ 3.7)$. The radial profiles of the triple correlation of in-plane velocity fluctuations normalized by $U_{c}^{3}$ are shown in figure 16. To compare the trends we also show the respective profiles for a single-phase jet (Darisse et al. 2012) and a bubble plume (Lai \& Socolofsky 2019). The profiles extracted from the two references are multiplied by a factor for visual comparison (see legends in figure 16).

Other than $\left\langle u_{1}^{3}\right\rangle$, all triple-correlation profiles for the particle plume show trends similar to a turbulent single-phase jet. The triple-correlation profiles of the first two terms $\left(\left\langle u_{1}^{3}\right\rangle / U_{c}^{3}\right.$ and $\left.\left\langle u_{1} u_{2}^{2}\right\rangle / U_{c}^{3}\right)$ for the bubble plume in Lai \& Socolofsky (2019) are significantly different from the single-phase jet and our particle plume, and they show 10-20 times larger magnitude compared to our particle plume results (see figure $16 a, b)$. The bubble plume shows a positive $\left\langle u_{1}^{3}\right\rangle$ near the centreline as opposed to the negative $\left\langle u_{1}^{3}\right\rangle$ observed near the core of the particle plume, due to the opposite skewness of their $u_{1}$ distributions discussed earlier (see figure 10a).

Based on the triple-correlation terms computed above, we estimate the axial and radial transport of $k$ in figures $17(a)$ and 17(b), respectively. Since the TKE in the particle plume is primarily contributed by the axial stress, $\left\langle u_{1}^{2}\right\rangle$, these two profiles are nearly identical to those in figure $16(a, c)$. These profiles are fitted to two shape functions (see appendix A) and are shown as solid lines in figures 17(a) and 17(b), respectively. We use them for estimating the TKE budget in $\S 3.7$.

\subsection{Mean square gradients of velocity fluctuations}

To test the conditions of local isotropy and local axisymmetry (George \& Hussein 1991), we compute the in-plane derivatives of all three components of velocity fluctuations employing the second-order-accurate central difference scheme. Table 3 and figure 18 show the second moments of axial $\left(\partial / \partial x_{1}\right)$ and radial $\left(\partial / \partial x_{2}\right)$ derivatives of all three components of velocity. These moments clearly do not satisfy the conditions of local isotropy (e.g. $\left\langle\left(\partial u_{1} / \partial x_{1}\right)^{2}\right\rangle \neq\left\langle\left(\partial u_{2} / \partial x_{2}\right)^{2}\right\rangle$ and $\left\langle\left(\partial u_{1} / \partial x_{2}\right)^{2}\right\rangle \neq$ $\left.2\left\langle\left(\partial u_{1} / \partial x_{1}\right)^{2}\right\rangle\right)$ nor of local axisymmetry (e.g. $\left.\left\langle\left(\partial u_{3} / \partial x_{1}\right)^{2}\right\rangle \neq\left\langle\left(\partial u_{2} / \partial x_{1}\right)^{2}\right\rangle\right)$.

The violation of local axisymmetry was also observed for bubble plumes (Lai \& Socolofsky 2019), suggesting that this is a common characteristic of multiphase plume turbulence contrary to single-phase jets.

Computing the true viscous dissipation rate (stress power) requires all nine components of the velocity gradient tensor and each component has to be adequately 

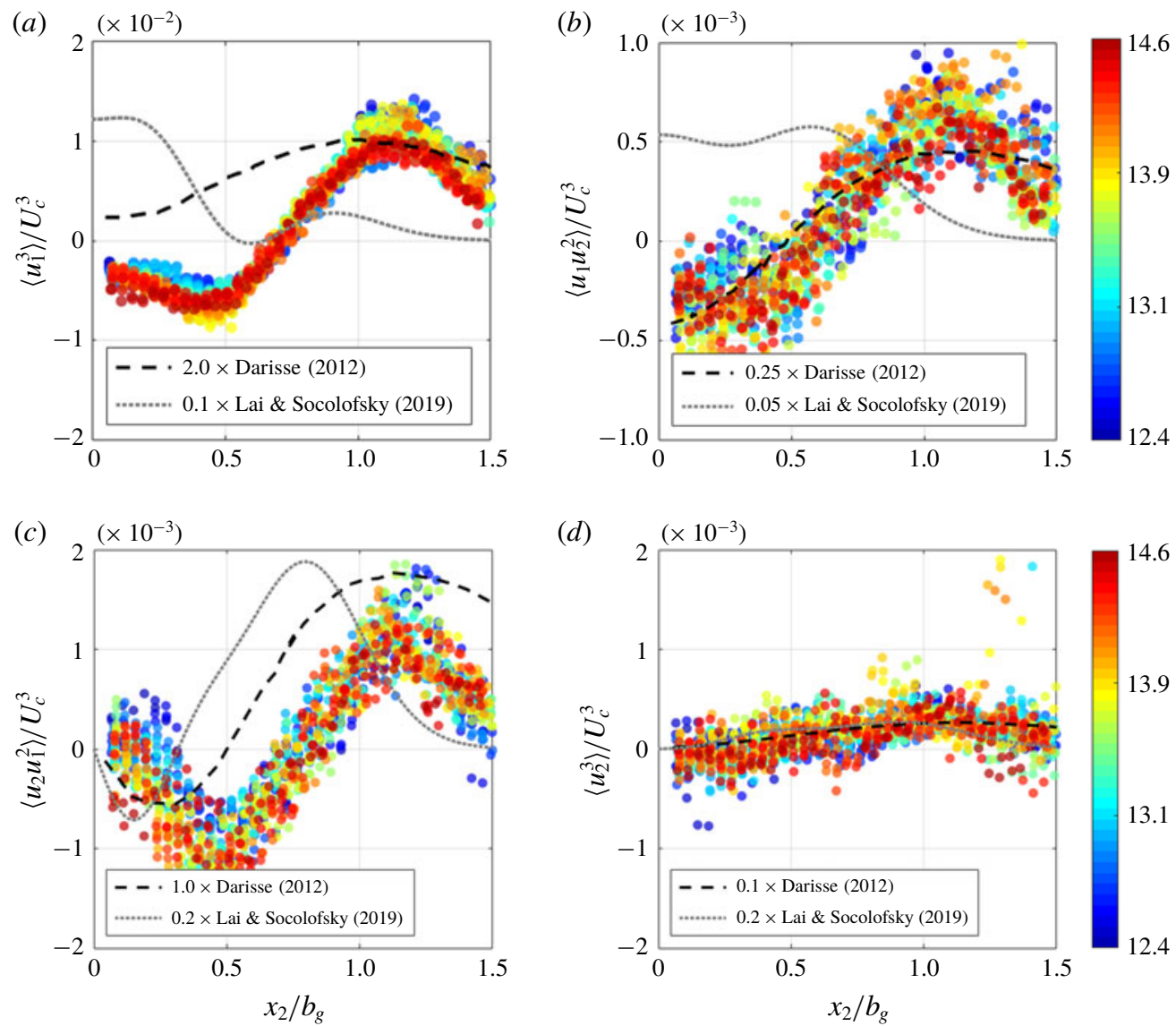

FIGURE 16. Normalized radial profiles of triple correlations $(a, b)$ axial transport $\left(\left\langle u_{1} u_{i}^{2}\right\rangle\right)$ and $(c, d)$ radial transport $\left(\left\langle u_{2} u_{i}^{2}\right\rangle\right)$ of in-plane components of turbulent kinetic energy in a particle plume at various axial locations $\left(x_{1} / d_{0}\right)$ indicated by the colourbar compared with a single-phase jet (Darisse et al. 2012) and a bubble plume (Lai \& Socolofsky 2019).

resolved, which is not easy to achieve experimentally. Instead, we compute the pseudo-dissipation rate $\langle\epsilon\rangle$ (Pope 2000),

$$
\begin{aligned}
\langle\epsilon\rangle= & v\left[\left\langle\left(\frac{\partial u_{1}}{\partial x_{1}}\right)^{2}\right\rangle+\left\langle\left(\frac{\partial u_{1}}{\partial x_{2}}\right)^{2}\right\rangle+\left\langle\left(\frac{\partial u_{1}}{\partial x_{3}}\right)^{2}\right\rangle\right. \\
& +\left\langle\left(\frac{\partial u_{2}}{\partial x_{1}}\right)^{2}\right\rangle+\left\langle\left(\frac{\partial u_{2}}{\partial x_{2}}\right)^{2}\right\rangle+\left\langle\left(\frac{\partial u_{2}}{\partial x_{3}}\right)^{2}\right\rangle \\
& \left.+\left\langle\left(\frac{\partial u_{3}}{\partial x_{1}}\right)^{2}\right\rangle+\left\langle\left(\frac{\partial u_{3}}{\partial x_{2}}\right)^{2}\right\rangle+\left\langle\left(\frac{\partial u_{3}}{\partial x_{3}}\right)^{2}\right\rangle\right] .
\end{aligned}
$$

Here, $v=1.02 \times 10^{-6} \mathrm{~m}^{2} \mathrm{~s}^{-1}$ is the kinematic viscosity of saline water at $23^{\circ} \mathrm{C}$. In our calculations all but the three out-of-plane derivative terms in (3.3) are computed directly from our SPIV data, and the missing out-of-plane mean-square derivatives 

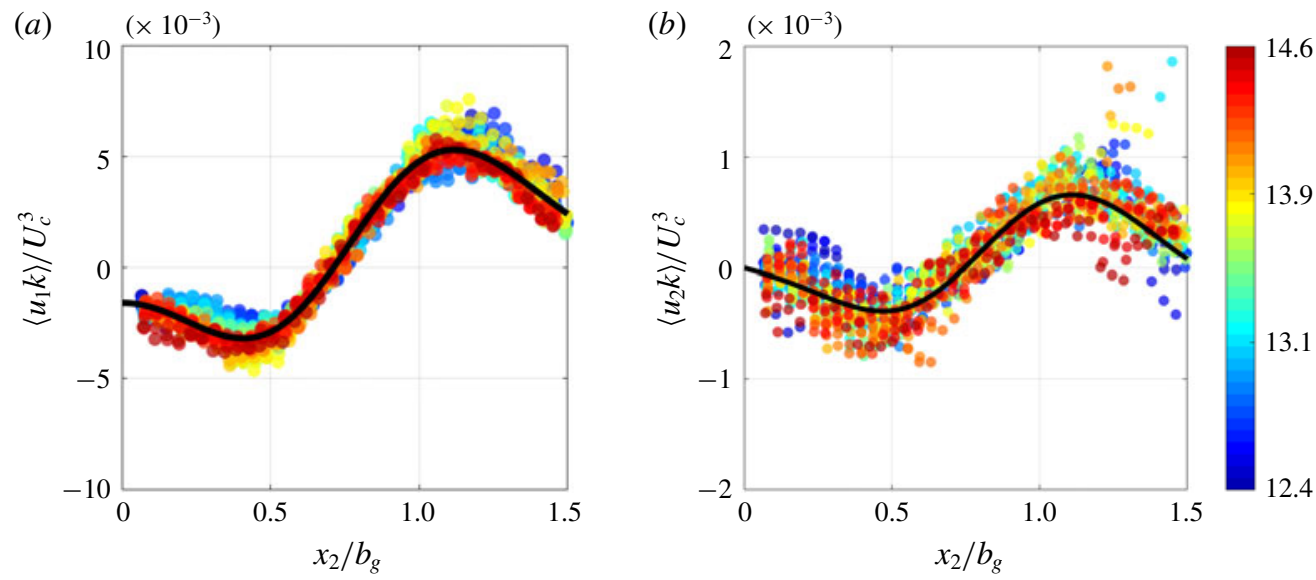

FIGURE 17. Radial profiles of non-dimensional (a) axial $\left(\left\langle u_{1} k\right\rangle / U_{c}^{3}\right)$ and $(b)$ radial $\left(\left\langle u_{2} k\right\rangle / U_{c}^{3}\right)$ transport of turbulent kinetic energy at various axial locations $\left(x_{1} / d_{0}\right)$ indicated by the colourbar.
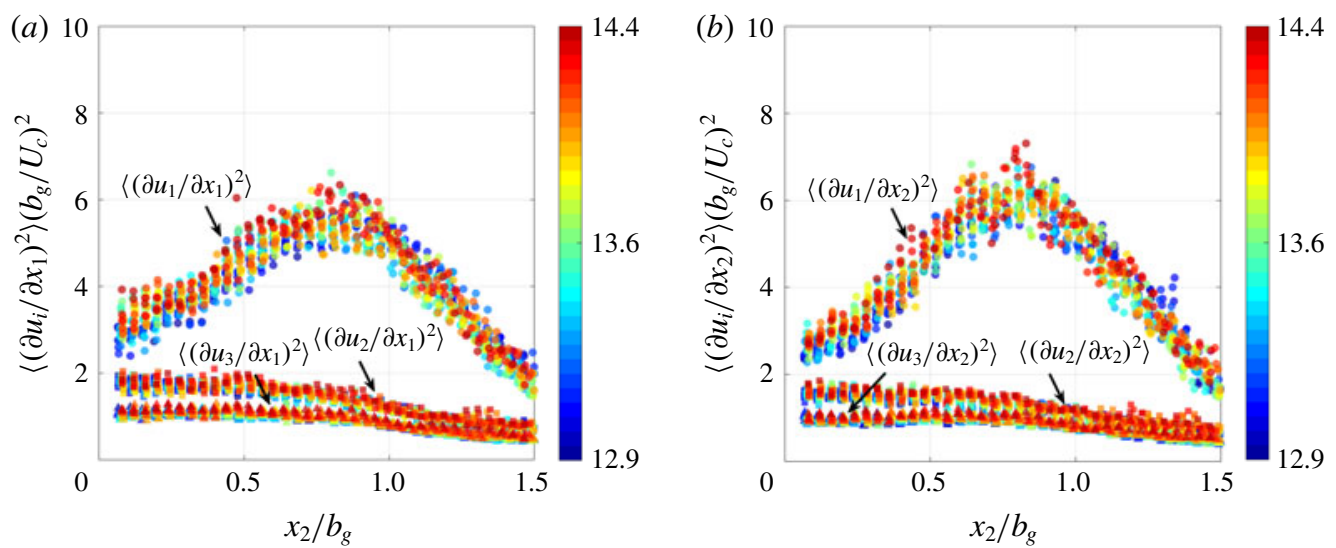

FIGURE 18. Radial profiles of non-dimensional (a) axial $\left(\left\langle u_{1} k\right\rangle / U_{c}^{3}\right)$ and $(b)$ radial $\left(\left\langle u_{2} k\right\rangle / U_{c}^{3}\right)$ transport of turbulent kinetic energy at various axial locations $\left(x_{1} / d_{0}\right)$ indicated by the colourbar.

$\left(\left\langle\left(\partial u_{i} / \partial x_{3}\right)^{2}\right\rangle\right)$ are replaced by $\left\langle\left(\partial u_{i} / \partial x_{2}\right)^{2}\right\rangle$. The mean pseudo-dissipation rate $\langle\epsilon\rangle$ across the plume is shown for various axial locations in figure 19.

To assess the accuracy in the dissipation calculation, we adopt a method based on conservation of total kinetic energy for multiphase plume in a Lagrangian framework as outlined in Lai \& Socolofsky (2019). In essence, this method estimates the balance of the total kinetic energy as production minus dissipation

$$
\iiint_{V} \frac{D}{D t}(\langle K\rangle+\langle k\rangle) \mathrm{d} V=-f_{c} \iiint_{V}\langle\epsilon\rangle \mathrm{d} V+\iiint_{V} \frac{\Delta \rho}{\rho}\left\langle U_{1}\right\rangle g \mathrm{~d} V,
$$

and calculates the correction factor $f_{c}$ to balance the two sides. The mean kinetic energy, $\langle K\rangle \approx\left\langle U_{1}\right\rangle^{2} / 2$ because $U_{1} \gg U_{2}, U_{3}$. This equation can be simplified in the 


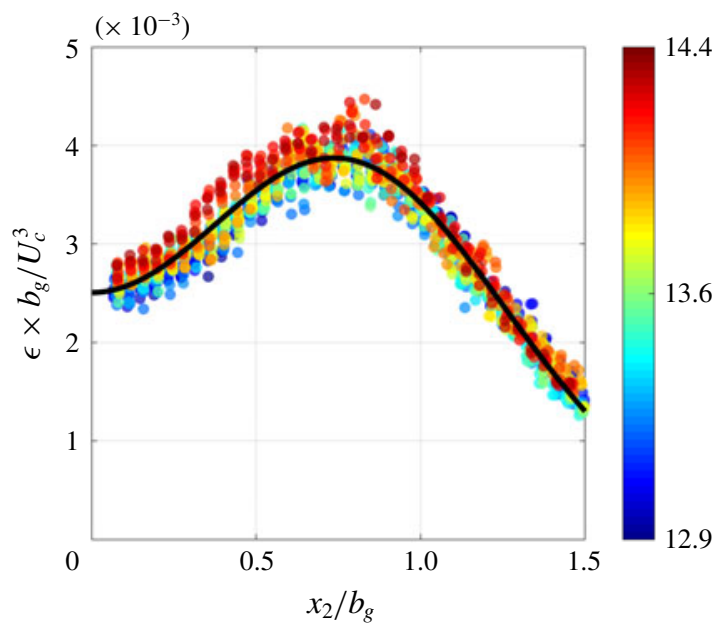

FIGURE 19. Radial profiles of non-dimensional mean dissipation rate at various axial locations $\left(x_{1} / d_{0}\right)$ indicated by the colourbar.

Term in (3.4)

Full form

Similarity form

Value

Flux of $K, I_{1} \quad 2 \pi \int_{0}^{\infty}\left\langle U_{z}\right\rangle\langle K\rangle r \mathrm{~d} r$

$2 \pi \int_{0}^{\infty} \eta \mathrm{e}^{-3 \eta^{2}} \mathrm{~d} \eta$

Flux of $k, I_{2} \quad 2 \pi \int_{0}^{\infty}\left\langle U_{z}\right\rangle\langle k\rangle r \mathrm{~d} r$

$2 \pi \int_{0}^{\infty} \eta \mathrm{e}^{-\eta^{2}} F_{4}(\eta) \mathrm{d} \eta$

0.095-0.105

Dissipation, $I_{3}$

$$
2 \pi \int_{0}^{\infty}\langle\epsilon\rangle r \mathrm{~d} r
$$

$2 \pi \int_{0}^{\infty} \eta F_{5}(\eta) \mathrm{d} \eta$

$0.021-0.027$

$K \approx U_{1}^{2} / 2$

$k=\left(u_{1}^{2}+u_{2}^{2}+u_{3}^{2}\right) / 2$

TABLE 4. Simplified similarity expressions and values of various integrals used in (3.5). The shape functions $F_{4}(\eta)$ and $F_{5}(\eta)$ are summarized in appendix A.

cylindrical coordinate system $(r, \theta, z)$ by invoking steady state as follows. The lefthand side of the equation is computed using the shape functions of the mean radial profiles for $\left\langle U_{1}\right\rangle$ and $\langle k\rangle$ (see $\S \S 3.1$ and 3.2). The first term on the right-hand side is obtained by using $\langle\epsilon\rangle$ at multiple axial locations. The last term in (3.4) refers to the kinetic energy production due to the particle buoyancy flux and equals $C F_{0} \Delta x_{1}$, where the prefactor $C=1+\lambda^{2} / \pi \lambda^{2} \sqrt{4 / \gamma} \int_{0}^{\infty} 2 \pi \eta \mathrm{e}^{-\left(2 \lambda^{2}+1 / \lambda^{2}\right)} \eta^{2} \mathrm{~d} \eta$ depends on $\lambda=\left\langle b_{\phi}\right\rangle /\left\langle b_{g}\right\rangle$ and the momentum amplification factor $\gamma$. Based on $\lambda=0.6$ and $\gamma=1.2$, we obtain $C=1.44$. The simplified equation for a volume bounded by two axial locations $x_{1}$ and $x_{2}$ is written as

$$
\left[\left(0.5 I_{1}+I_{2}\right)\left(b_{g}^{2} U_{c}^{3}\right)\right]_{x_{1}}^{x_{2}}=f_{c} \int_{z_{1}}^{z_{2}} I_{3} b_{g} U_{c}^{3} \mathrm{~d} x+1.44 F_{0} \Delta x_{1} .
$$

Here, $I_{1}, I_{2}$ and $I_{3}$ are the axisymmetric surface integrals $\left(2 \pi \int_{0}^{\infty}(). r \mathrm{~d} r\right)$ of the respective terms inside the volume integral in (3.4) (summarized in table 4).

We test the balance of (3.5) at multiple axial locations separated by $1.5 b_{g}$ and have found $f_{c}$ to vary between 2.8 and 5.1 with a mean value of 4.0. This method 
estimates the amount by which $\langle\epsilon\rangle$ is underestimated by our evaluation of (3.6), but it is unable to correct $\langle\epsilon\rangle$ locally along the radius of the plume. Given such limitation, $\langle\epsilon\rangle$ presented here must be interpreted as order of magnitude estimates. We use the corrected dissipation profile only for a qualitative assessment of the TKE budget discussed in the following section.

\subsection{Turbulent kinetic energy budget}

In the cylindrical coordinate system, the time-averaged transport equation for $k$ of an axisymmetric, steady, turbulent plume without swirl is (Kataoka \& Serizawa 1989)

$$
\begin{aligned}
0=-\overbrace{\left.\left(U_{r}\right\rangle \frac{\partial k}{\partial r}+\left\langle U_{z}\right\rangle \frac{\partial k}{\partial z}\right)}^{A} & \overbrace{-\left(\frac{1}{r} \frac{\partial\left\langle u_{r} k\right\rangle}{\partial r}+\frac{\partial\left\langle u_{z} k\right\rangle}{\partial z}\right)}^{T} \\
& \overbrace{\frac{1}{\rho}\left(\frac{1}{r} \frac{\partial\left\langle u_{r} p\right\rangle}{\partial r}+\frac{\partial\left\langle u_{z} p\right\rangle}{\partial z}\right)}^{T_{p}} \\
& \overbrace{\left(\left\langle u_{z}^{2}\right\rangle \frac{\partial\left\langle U_{z}\right\rangle}{\partial z}+\left\langle u_{r}^{2}\right\rangle \frac{\partial\left\langle U_{r}\right\rangle}{\partial r}+\frac{\left\langle U_{r}\right\rangle\left\langle u_{\theta}^{2}\right\rangle}{r}+\left\langle u_{z} u_{r}\right\rangle\left(\frac{\partial\left\langle U_{z}\right\rangle}{\partial r}+\frac{\partial\left\langle U_{r}\right\rangle}{\partial z}\right)\right)}^{P_{s}} \\
& -\langle\epsilon\rangle \\
& +P_{b} .
\end{aligned}
$$

In addition to the terms already present in single-phase jets/plumes ( $A$, advection; $P_{s}$, shear-production; $T$, turbulent transport; and $T_{p}$, pressure transport), a multiphase plume has another source term $\left(P_{b}\right)$ that represents the interfacial energy transfer at the boundaries between the dispersed phase and the surrounding fluid. Obtaining the complete TKE budget for a particle plume is challenging for multiple reasons. First, it is difficult to accurately measure experimentally the mean dissipation rate $\langle\epsilon\rangle$ because of the under-resolved velocity gradients (as seen in $\S 3.6$ ). Second, there is no existing model for pressure-velocity correlation in multiphase flow turbulence, and therefore the pressure transport term $\left(T_{p}\right)$ requires an assumption. Finally, the interfacial energy transfer $\left(P_{b}\right)$ is the most complex and inaccessible term, and it requires the local distribution of particles and their relative velocity with the surrounding fluid (Santarelli, Rouseel \& Frohlich 2016). In an ideal scenario with all other terms measured correctly, the term $P_{b}$ can be sought as the closing term of (3.6), such that $P_{b}=\langle\epsilon\rangle-\left(A+P_{s}+T+T_{p}\right)$. We will use this method to estimate $P_{b}$ from our data.

We directly compute $A, P_{s}$ and $T$ from our experimental data. The expressions for these in terms of similarity variables are summarized in appendix B. Each plot contains an error band corresponding to the $95 \%$ confidence interval in the constituent fits. Each uncertainty band is computed via the rule of uncertainty propagation described in Charonko \& Prestridge (2017). The pressure transport term, $T_{p}$, not directly available from experiment, is replaced by Lumley's model $T_{p}=-2 / 5 T$, 

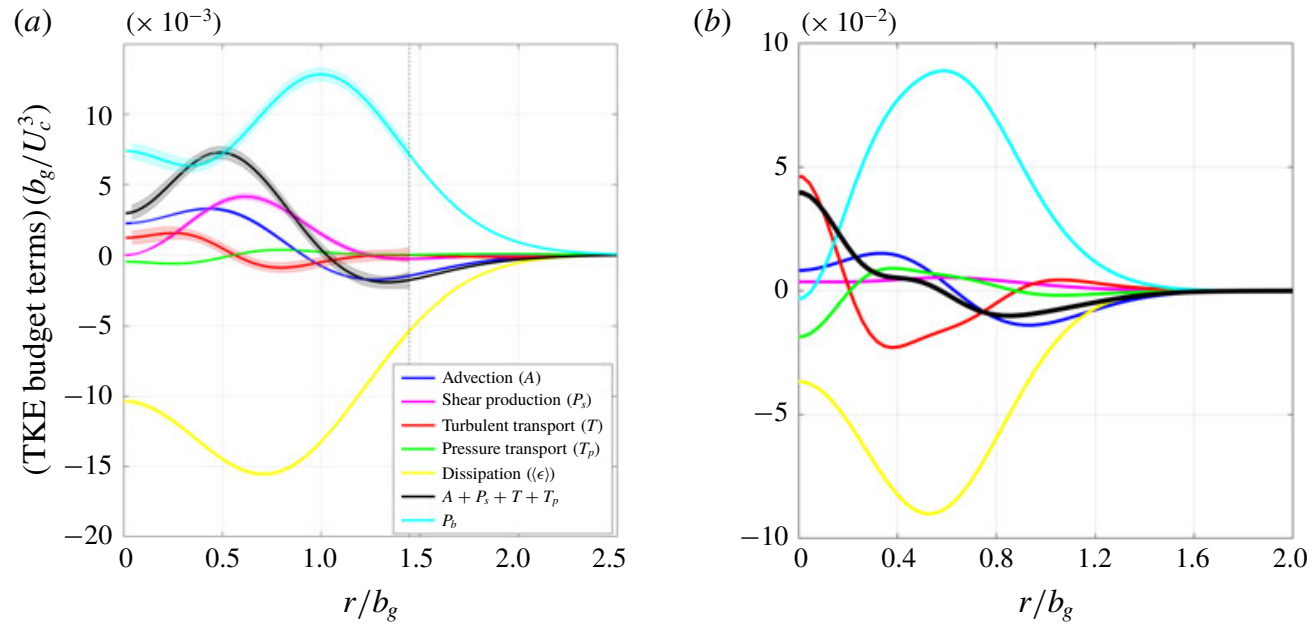

FIGURE 20. Various terms in the turbulent kinetic energy budget for $(a)$ a particle plume and (b) a bubble plume reproduced from Lai \& Socolofsky (2019). The error band in each term (shown up to the extent of measurement window) represents uncertainty in computing the term.

widely used for single-phase turbulence (Lumley 1978). These four terms and the mean dissipation rate $(\langle\epsilon\rangle)$ multiplied by the correction factor $f_{c}=4$ are shown in figure $20(a)$. The remaining terms of the balance equation are the interfacial energy transfer $\left(P_{b}\right)$ from particles to fluid and out-of-balance experimental error (known as OOBE). The budget terms for a bubble plume from Lai \& Socolofsky (2019) are reproduced in figure 20(b) for comparison. Each budget term is normalized by $U_{c}^{3} / b_{g}$; the order of magnitude difference in the two budgets arises because of the difference in the downstream measurement locations with respect to the source, leading to the differences in $U_{c}$ and $b_{g}$ (see figure $7 a, b$ ). Below we discuss the comparison of the TKE budget for the two multiphase plumes in light of previous experiments on a single-phase jet (Lai \& Socolofsky 2018) and a variable-density single-phase (VDSP) jet (Charonko \& Prestridge 2017). The qualitative summary of this comparison is provided in table 5.

The shape of the advection $(A)$ profiles are very similar across all four plumes with a positive central lobe inside the plume core. The shear production term $\left(P_{s}\right)$ in the particle plume increases from zero at the centreline to a dominant peak at approximately $r / b_{g}=0.65$ near the edge of the plume. This behaviour strongly resembles a single-phase jet both in shape and relative magnitude. The $P_{s}$ profile in the VDSP jet also matches this trend, except that a negative $P_{s}$ is observed at the jet centreline. By contrast, the bubble plume shows almost negligible $P_{s}$ across the radius of the plume. The turbulent transport term $(T)$ in the particle plume exhibits a positive peak near the centreline and a negative lobe near the edge of the plume. The bubble plume and the VDSP jet are similar, but with a larger peak in the former. The single-phase jet does not match the others, i.e. near the centreline the turbulent transport is consistently observed to be negative or marginally positive (Darisse, Lemay \& Benaïssa 2015).

Because the energy production at the two-phase boundaries, $P_{b}$, is computed indirectly, the peak location in $P_{b}$ is sensitive to the experimental error in $\langle\epsilon\rangle$. 
TKE budget term

Advection, $A$

Shear production, $P_{s}$

Turbulent transport, $T$

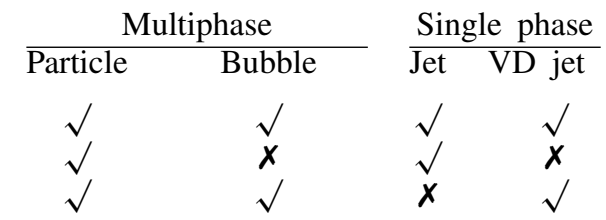

$\boldsymbol{X}$ : dissimilar profile shapes

TABLE 5. Qualitative comparison summary of three TKE budget terms in our particle plume and those in a bubble plume (Lai \& Socolofsky 2019), a single-phase jet (Darisse et al. 2015; Lai \& Socolofsky 2018) and a VDSP jet (Charonko \& Prestridge 2017).

Despite this limitation, it is worth noting that in both particle and bubble plumes, the sum $\left(A+T+P_{s}+T_{p}\right)$ is significantly smaller than the mean dissipation rate outside the plume core $\left(r>0.5 b_{g}\right)$. This results in the TKE budget being an approximate balance between $P_{b}$ and $\langle\epsilon\rangle$ lending further credibility to our results.

\subsection{Two-point correlation and energy spectra}

We obtain the two-point statistics from the spatial autocorrelation function $R_{11}$ computed along the axial measurements of axial velocity fluctuations, $u_{1}$. Figure 21 shows $R_{11}$ inside the plume core $\left(x_{2} / b_{g}<0.5\right)$ at various radial locations (shaded circles) and their mean $\bar{R}_{11}$ (red circles). The horizontal axis is normalized by the particle diameter $d_{p}$. We estimate the Taylor microscale $\left(\lambda_{f}\right)$ of this flow by fitting an osculating parabola, $1-\left(x_{1}^{2} / \lambda_{f}^{2}\right)$ (Pope 2000) to the first four points of $\bar{R}_{11}$ (see solid line in figure 21), yielding $\lambda_{f}=1.3 d_{p}$, which is of the order of the particle size. The Kolmogorov scale obtained based on $\lambda_{f}$ is approximately $0.1 \mathrm{~mm}$, which suggests that our velocity gradient measurements are 'coarse-grained' and are subject to the inherent spatial averaging over the PIV subwindow. The Taylor microscale, $\lambda_{f}$, is related to the mean square velocity gradient in homogeneous isotropic turbulence (Pope 2000) and we use this relation to approximate

$$
\left\langle\left(\frac{\partial u_{1}}{\partial x_{1}}\right)^{2}\right\rangle \approx \frac{2\left\langle u_{1}^{2}\right\rangle}{\lambda_{f}^{2}} .
$$

Based on this method, $\left\langle\left(\partial u_{1} / \partial x_{1}\right)^{2}\right\rangle\left(b_{g} / U_{c}\right)^{2}$ at $x_{2}=0$ is $3.2 \pm 0.6$ and at $x_{2}=0.5 b_{g}$ is $5.8 \pm 0.6$. Within the uncertainty bounds, these values match those obtained from direct computation of gradients in $\$ 3.6$ (see figure $18 a$ ).

Risso (2018) suggests that the inter-scale energy transfer in bubble-induced agitation (known as BIA) is quite different from the classical picture of shear-induced turbulence (known as SIT). As a first step toward understanding the energy cascade in particle-plume turbulence, we generate the one-dimensional energy spectrum $\left(E_{11}\right)$ from the autocorrelation function $\bar{R}_{11}$ (Pope 2000). The normalized spectra for our particle plume is compared with experimental results (Riboux et al. 2010) and DNS simulations (Lai et al. 2018) in figure 22(a). The result shows striking similarity in the spectra between homogeneous bubble swarms (both experiment and DNS simulation) and our particle plume; for wavenumbers smaller than $1 / \lambda_{f}$, all follow a power law close to $\kappa^{-3}$ similar to the earlier prediction (Lance \& Bataille 1991). Beyond $\kappa_{1}>1 / \lambda_{f}$, both experimental results (ours and Riboux et al. (2010)) show different 


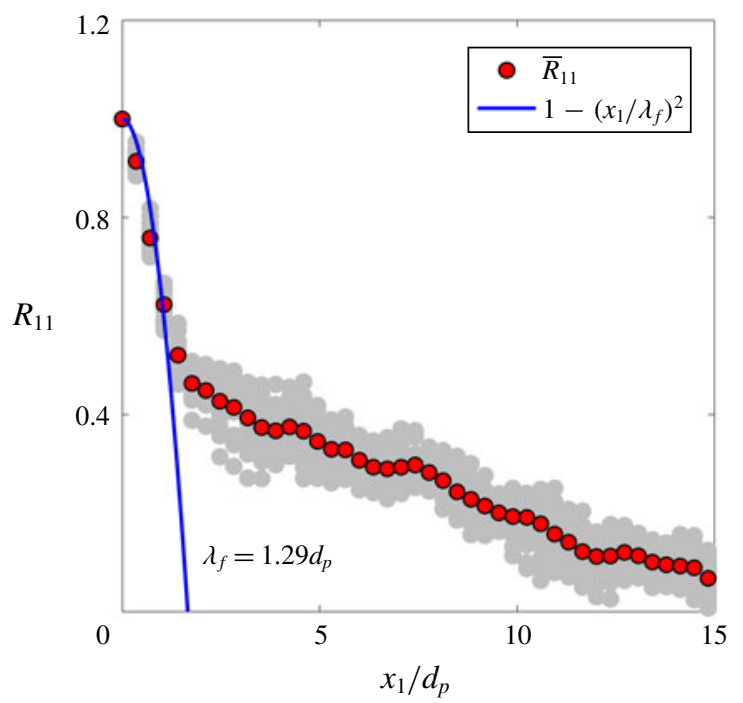

FIGURE 21. Spatial autocorrelation $R_{11}$ along longitudinal (axial) direction of the flow. The shaded circles show autocorrelation at various radial location $\left(x_{2} / b_{g}<0.5\right)$, the filled circles are their mean. The solid line is an osculating parabola fit to the first four data points in $\bar{R}_{11}$.
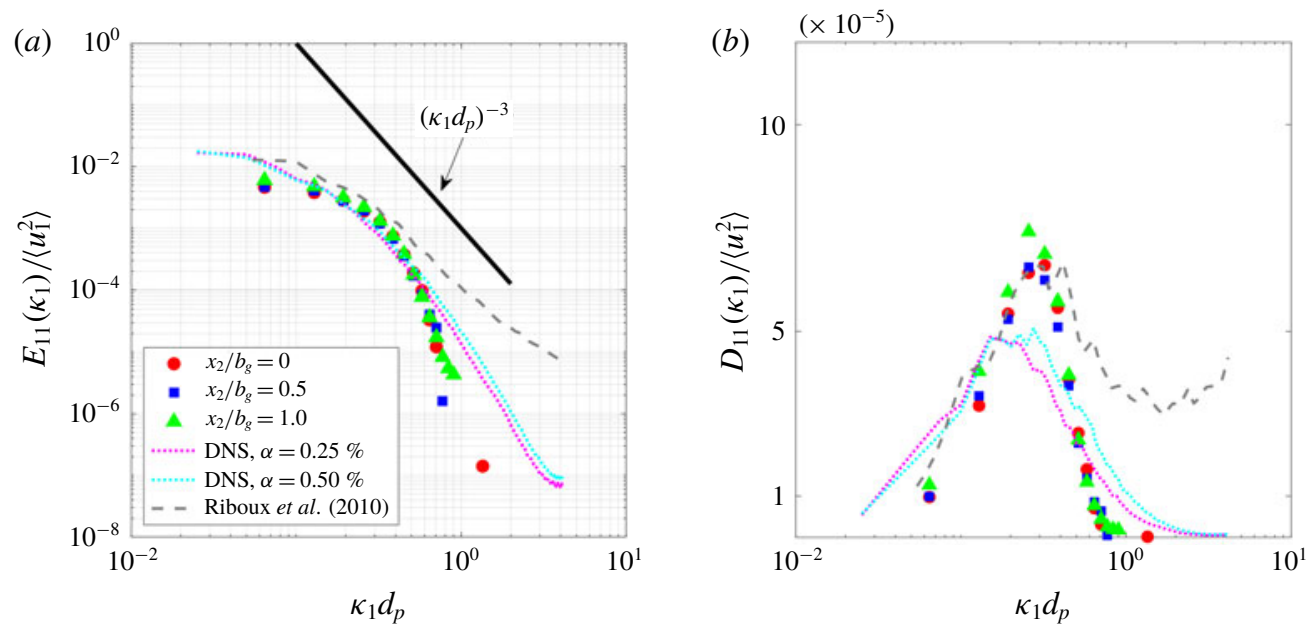

FIGURE 22. Comparison of $(a)$ one-dimensional power spectra $E_{11}\left(\kappa_{1}\right)$ of axial velocity fluctuations and $(b)$ one-dimensional dissipation spectra in particle plume with earlier studies related to homogeneous bubble swarms (experiment, (Riboux et al. 2010); DNS simulation, Lai et al. (2018)). The horizontal axis is normalized by the wavenumber corresponding to respective particle diameter $\left(d_{p}\right)$.

slopes than the DNS results, which can be attributed to experimental uncertainty in resolving velocity fluctuations below $\lambda_{f}$. Nonetheless, this result is valuable and it suggests power law universality of multiphase flow turbulence which is quite different from that for single-phase turbulent shear-flows. 
The one-dimensional dissipation spectra $D_{11}\left(\kappa_{1}\right)=2 \nu \kappa^{2} E_{11}\left(\kappa_{1}\right)$ is shown in figure 22(b). Quite strikingly, the peaks of dissipation for our data and bubble plume/swarms reside at $\kappa_{1} d_{p} \approx 0.2-0.4$. This indicates that in both bubble- and particle-laden turbulence, production and dissipation occur near the scale of particle size. This lack of scale separation was first postulated for homogeneous bubbly flows in Lance \& Bataille (1991) and is observed in direct numerical simulations reported in Lai et al. (2018).

\section{Conclusions}

We report an experimental study characterizing the turbulence inside a heavy particle plume descending under gravity within a salt-water solution. We measure the three components (i.e. 2D3C) of the interstitial fluid velocity and the spatial distribution of particles in the central plane of the plume using refractive-indexmatched stereoscopic particle image velocimetry. Below we summarize our key findings primarily in the light of the results for a bubble plume (Lai \& Socolofsky 2019).

The induced liquid flow inside the plume evolves with the mean flow characteristics of a bubble plume (Lai \& Socolofsky 2019), that show Gaussian mean axial velocity $\left(\left\langle U_{1}\right\rangle\right)$ profile. The radial profiles of mean particle number density are also Gaussian with a half-width $b_{\phi}$ equals to $0.56 b_{g}$, where $b_{g}$ is the Gaussian half-width of the mean streamwise velocity profile in the fluid.

The turbulence inside the plume is highly anisotropic with maximum streamwise turbulence intensity measuring up to 2.7 times that of the other two components, a result consistent with that observed in a bubble plume. The p.d.f. of the axial velocity fluctuation $\left(u_{1}\right)$ at the centreline of the plume is skewed so that the strongest events are in the direction opposite to mean flow. This behaviour is strikingly opposite to that observed previously for a bubble plume and a homogeneous swarm of bubbles. The turbulent kinetic energy and the in-plane shear-stress peak near $\approx 0.75 b_{g}$, locating the shear layer slightly outside the edge of the particle core $\left(b_{\phi}\right)$. Although these quantities for a bubble plume in Lai \& Socolofsky (2019) peaked at a similar location $\left(\approx 0.7 b_{g}\right)$, the location of the shear layer with respect to their bubble-core $\left(b_{\phi}\right)$ remains unknown.

Another distinction between the particle and the bubble plume is observed in the fluid-shear production term, $P_{s}$, in the TKE budget. While the shear production in the bubble plume is negligible across the plume compared to the other terms, we observe a distinct peak in $P_{s}$ at the shear layer region of a particle plume, resembling a singlephase jet.

The other TKE terms including advection, $A$, and the turbulent transport, $T$, in the two flows exhibit similar profiles. Further, we show that the turbulent transport, $T$, term follows similar qualitative profiles for both plumes and also an earlier reported variable-density single-phase jet (Charonko \& Prestridge 2017), all of which differ from a typical single-phase jet. The difference in the relative magnitude in $T$ at the centreline between the two plumes is attributed to the difference in their third moment (skewness) of the axial velocity fluctuations.

Despite the above differences inside the core, both particle and bubble plumes show qualitatively that the TKE production by the particles, $P_{b}$, approximately balances the mean dissipation rate, $\langle\epsilon\rangle$, away from the centreline. Further, the one-dimensional spectrum in the particle plume exhibits the -3 power law consistent with bubble plume and homogeneous swarms of bubbles. These two results support the notion that there is a lack of separation between the scales of production and dissipation in multiphase turbulent flows like ours. 


$\begin{array}{lcccc}\text { Function }\left(F_{i}(\eta)\right) & \text { Parameter } & f_{1} & f_{2} & f_{3} \\ F_{1} & \left\langle u_{z}^{2}\right\rangle / U_{c}^{2} & 0.069 & 0.74 & 0.63 \\ F_{2} & \left\langle u_{r}^{2}\right\rangle / U_{c}^{2} & 0.011 & 0.57 & 1.00 \\ F_{3} & \left\langle u_{\theta}^{2}\right\rangle / U_{c}^{2} & 0.009 & 0.62 & 0.84 \\ F_{4} & k / U_{c}^{2} & 0.045 & 0.71 & 0.68 \\ F_{5} & \langle\epsilon\rangle b_{g} / U_{c}^{3} & 0.004 & 0.76 & 0.72\end{array}$

TABLE 6. Coefficients of double-Gaussian function in (A 1) used to fit various parameters measured in the experiment.

\section{Acknowledgements}

This research was supported by the National Science Foundation (grant no. OCE1334788) and by The Gulf of Mexico Research Initiative. The authors also thank M. Heath for his help in the design of the experimental set-up and data collection.

\section{Declaration of interests}

The authors report no conflict of interest.

\section{Appendix A.}

The three components of normal stresses, the turbulent kinetic energy $(k)$ and the mean dissipation rate $\langle\epsilon\rangle$ are fitted into a shape preserving double-Gaussian curve, given in (A 1) as follows:

$$
F_{i}(\eta)=f_{1}\left[\exp \left(-\left(\frac{\eta-f_{3}}{f_{2}}\right)^{2}\right)+\exp \left(-\left(\frac{\eta+f_{3}}{f_{2}}\right)^{2}\right)\right] .
$$

All terms are expressed in similarity variable $\eta=r / b_{g}$.

The in-plane shear-stress is fitted into a polynomial-Gaussian function in (A 2) as follows:

$$
S=\left\langle u_{z} u_{r}\right\rangle / U_{c}^{2}=\left(0.0124 \eta+0.0286 \eta^{3}-0.0113 \eta^{5}\right) \exp \left(-1.47 \eta^{2}\right) .
$$

The axial and radial transport of the turbulent kinetic energy $(k)$ are fitted into the polynomial-Gaussian functions in (A 3) and (A 4), respectively, as

$$
\begin{gathered}
T_{1}=\left\langle u_{z} k\right\rangle / U_{c}^{3}=\left(-0.0016-0.0252 \eta^{2}-0.0181 \eta^{4}+0.0746 \eta^{6}\right) \exp \left(-2.62 \eta^{2}\right), \\
T_{2}=\left\langle u_{r} k\right\rangle / U_{c}^{3}=\left(-0.0008 \eta 0.0048 \eta^{3}-0.0139 \eta^{5}-0.0049 \eta^{7}\right) \exp \left(-1.76 \eta^{2}\right) .
\end{gathered}
$$

\section{Appendix B.}

Expressions for the advection, $A$, shear-production, $P_{s}$, and turbulent transport, $T$, terms in cylindrical coordinate system in the turbulent kinetic energy budget are summarized below. Each term is expressed in terms of the similarity variable $\eta=r / b_{g}$.

(i) Advection term

$$
\begin{gathered}
A=-\left[\left\langle U_{z}\right\rangle \frac{\partial k}{\partial z}+\left\langle U_{r}\right\rangle \frac{\partial k}{\partial r}\right], \\
A \times \frac{b_{g}}{U_{c}^{3}}=\left[\frac{2}{3} F_{4}(\eta)+\eta \frac{\mathrm{d} F_{4}(\eta)}{\mathrm{d} \eta}\right] \beta \mathrm{e}^{-\eta^{2}}-\left[G(\eta) \frac{\mathrm{d} F_{4}(\eta)}{\mathrm{d} \eta}\right] .
\end{gathered}
$$


(ii) Production term

$$
\begin{aligned}
& P=-[\underbrace{\left\langle u_{z}^{2}\right\rangle \frac{\partial\left\langle U_{z}\right\rangle}{\partial z}}_{P_{1}}+\underbrace{\left\langle u_{r}^{2}\right\rangle \frac{\partial\left\langle U_{r}\right\rangle}{\partial r}}_{P_{2}}+\underbrace{\frac{\left\langle U_{r}\right\rangle\left\langle u_{\theta}^{2}\right\rangle}{r}}_{P_{3}}+\underbrace{\left\langle u_{z} u_{r}\right\rangle\left(\frac{\partial\left\langle U_{z}\right\rangle}{\partial r}+\frac{\partial\left\langle U_{r}\right\rangle}{\partial z}\right)}_{P_{4}}], \\
& P_{1} \times \frac{b_{g}}{U_{c}^{3}}=\frac{\beta}{3} F_{1}(\eta) \mathrm{e}^{-\eta^{2}}\left(1-6 \eta^{2}\right), \\
& P_{2} \times \frac{b_{g}}{U_{c}^{3}}=-F_{2}(\eta) \frac{\mathrm{d} G(\eta)}{\mathrm{d} \eta}, \\
& P_{3} \times \frac{b_{g}}{U_{c}^{3}}=-\frac{1}{\eta} F_{3}(\eta) G(\eta), \\
& P_{4} \times \frac{b_{g}}{U_{c}^{3}}=S(\eta)\left(2 \eta \mathrm{e}^{-\eta^{2}}+\frac{\beta}{3} G(\eta)+\eta \beta \frac{\mathrm{d} G(\eta)}{\mathrm{d} \eta}\right) .
\end{aligned}
$$

(iii) Turbulent transport

$$
\begin{gathered}
T=-\left[\frac{\partial\left\langle u_{z} k\right\rangle}{\partial z}+\frac{1}{r} \frac{\partial\left\langle u_{r} k\right\rangle}{\partial r}\right], \\
T \times \frac{b_{g}}{U_{c}^{3}}=\beta\left(T_{1}(\eta)+\eta \frac{\mathrm{d} T 1}{\mathrm{~d} \eta}\right)-\left(\frac{T_{2}(\eta)}{\eta}+\frac{\partial T_{2}(\eta)}{\partial \eta}\right) .
\end{gathered}
$$

\section{REFERENCES}

Almeras, E., Mathai, V., Lohse, D. \& Sun, C. 2017 Experimental investigation of the turbulence induced by a bubble swarm rising within incident turbulence. J. Fluid Mech. 825, 1091-1112.

Azimi, A. H., Zhu, D. Z. \& Rajaratnam, N. 2011 Effect of particle size on the characteristics of sand jets in water. J. Engng Mech. 137 (12), 822-834.

Azimi, A. H., ZHU, D. Z. \& RajaRATnAM, N. 2012 Computational investigation of vertical slurry jets in water. Intl J. Multiphase Flow 47, 94-114.

BAINES, P. \& SPARKS, R. 2005 Dynamics of giant volcanic ash clouds from supervolcanic eruptions. Geophys. Res. Lett. 32, L24808.

BAINES, P. G. 2008 Mixing in downslope flows in the ocean plumes versus gravity currents. Atmos.Ocean 46, 402-419.

Balachandar, S. \& Eaton, J. K. 2010 Turbulent dispersed multiphase flow. Annu. Rev. Fluid Mech. 42, 111-133.

Bombardelli, F. A., Buscaglia, G. C., Rehmann, C. R., Rincon, L. E. \& Garcia, M. H. 2007 Modeling and scaling of aeration bubble plumes: a two-phase ow analysis. J. Hydraul. Res. 45 (5), 617-630.

Bordoloi, A. D. \& VARIANO, E. A. 2017 Rotational kinematics of large cylindrical particles in turbulence. J. Fluid Mech. 815, 199-222.

Buscaglia, G. C., Bombardelli, F. A. \& Garcia, M. H. 2002 Numerical modeling of largescale bubble plumes accounting for mass transfer effects. Intl J. Multiphase Flow 28 (11), 1763-1785.

CARTEllier, A. \& Rivière, N. 2001 Bubble-induced agitation and microstructure in uniform bubbly flows at small to moderate particle Reynolds numbers. Phys. Fluids 13, 2165-2181.

Charonko, J.\& Prestridge, K. 2017 Variable-density mixing in turbulent jets with coflow. J. Fluid Mech. 825, 887-921. 
Clift, R., Grace, J. R. \& Weber, M. E. 1978 Bubbles, Drops and Particles. Academic Press, Inc.

DARISSE, A., Lemay, J. \& BEnAïsSA, A. 2012 LDV measurements of well converged third order moments in the far field of a free turbulent round jet. Exp. Therm. Fluid Sci. 44 (213), 823-833.

Darisse, A., Lemay, J. \& BenaÏssa, A. 2015 Budgets of turbulent kinetic energy, Reynolds stresses, variance of temperature fluctuations and turbulent heat fluxes in a round jet. J. Fluid Mech. 774, 95-142.

Duncan, B. B., Seol, D.-G. \& Socolofsky, S. A. 2009 Quantification of turbulence properties in bubble plumes using vortex identification methods. Phys. Fluids 21 (7), 075101.

Fraga, B. \& StOesser, T. 2016 Influence of bubble size, diffuser width, and flow rate on the integral behavior of bubble plumes. J. Geophys. Res. 121 (6), 3887-3904.

FreEth, S. 1987 The lake nyos disaster. Nature 325, 104-105.

GARCíA, C. M. \& GARCÍA, M. H. 2006 Characterization of flow turbulence in large-scale bubbleplume experiments. Exp. Fluids 41 (1), 91-101.

George, W. K. \& Hussein, H. 1991 Locally axisymmetric turbulence. J. Fluid Mech. 233, 1.

Gonzalez, R. C. \& Woods, R. E. 2007 Digital Image Processing, 3rd edn. Pearson.

Guazzelli, E. \& Morris, J. F. 2012 A Physical Introduction to Suspension Dynamics. Cambridge University Press.

Hall, N., Elenany, M., Zhu, D. Z. \& Rajaratnam, N. 2010 Experimental study of sand and slurry jets in water. J. Hydraul. Engng 136 (10), 727-738.

Huppert, H. E. \& Neufeld, J. A. 2014 The fluid mechanics of carbon dioxide sequestration. Annu. Rev. Fluid Mech. 46, 255-272.

KATAOKA, I. \& SERIZAWA, A. 1989 Basic equations of turbulence in gas-liquid two-phase flow. Intl J. Multiphase Flow 15, 843-855.

Lai, A. C. H., Chan, S. N., LAw, A. W.-K. \& AdAms, E. E. 2016 Spreading hypothesis of a particle plume. J. Hydraul. Engng 142 (12), 04016065.

Lai, C. C. K., Fraga, B., Chan, W. R. H.\& DodD, M. S. 2018 Energy cascade in a homogeneous swarm of bubbles rising in a vertical channel. Tech Rep. Center of Turbulence Research, Proceedings of Summer Program.

Lai, C. C. K. \& Socolofsky, S. A. 2018 Budgets of turbulent kinetic energy, Reynolds stresses, and dissipation in a turbulent round jet discharged into a stagnant ambient. Environ. Fluid Mech. 19, 1-29.

Lai, C. C. K. \& Socolofsky, S. A. 2019 The turbulent kinetic energy budget in a bubble plume. J. Fluid Mech. 865, 993-1041.

LANCE, M. \& BAtAille, J. 1991 Turbulence in the liquid phase of a uniform bubbly air-water flow. J. Fluid Mech. 222, 95-118.

Lee, J. H. W. \& CHU, V. H. 2003 Turbulent Jets and Plumes - A Lagrangian Approach. Kluwer Academic.

Loth, E. 2008 Drag of non-spherical solid particles of regular and irregular shape. Powder Technol. 182 (3), 342-353.

Lumley, J. L. 1978 Computational modeling of turbulent flows. Adv. Appl. Mech. 18, 123-176.

Mercado, J. M., Gomes, D. C., van Gils, D., Sun, C. \& Lohse, D. 2010 On bubble clustering and energy spectra in pseudo-turbulence. J. Fluid Mech. 650, 287-306.

Milgram, J. H. 1983 Mean flow in round bubble plumes. J. Fluid Mech. 133, 345-376.

Morton, B., TAYlor, G. \& TURner, J. 1956 Turbulent gravitational convection from maintained and instantaneous sources. Proc. R. Soc. Lond. A 234 (1), 1196.

Papanicolaou, P. N. \& List, E. J. 1988 Investigations of round vertical buoyant jets. J. Fluid Mech. 195, 341-395.

Pope, S. B. 2000 Turbulent Flows. Cambridge University Press.

Prakash, V. N., Mercado, J. M., Wijngaarden, E. M., Mancilla, E., Tagawa, Y., Lohse, D. \& Sun, C. 2016 Energy spectra in turbulent bubbly flows. J. Fluid Mech. 791, 174-190.

Riboux, G., Legendre, D. \& Risso, F. 2013 A model of bubble-induced turbulence based on large-scale wake interactions. J. Fluid Mech. 719, 362-387. 
Riboux, G., Risso, F. \& Legendre, D. 2010 Experimental characterization of the agitation generated by bubbles rising at high Reynolds number. J. Fluid Mech. 643, 509-539.

Risso, F. 2018 Agitation, mixing, and transfers induced by bubbles. Annu. Rev. Fluid Mech. 50, $25-48$.

Risso, F. \& Ellingsen, K. 2002 Velocity fluctuations in a homogeneous dilute dispersion of high-Reynolds-number rising bubbles. J. Fluid Mech. 453, 395-410.

SAntarelli, C. \& Rouseel, J. 2016 Budget analysis of the turbulent kinetic energy for bubbly flow in a vertical channel. Chem. Engng Sci. 141, 46-62.

Seol, D.-G., Duncan, B. B. \& Socolofsky, S. A. 2009 Measurements of behavioral properties of entrained ambient water in stratied bubble plume. ASCE J. Hydraul. Engng 135 (11), 983-988.

Simiano, M., Lakehal, D., Lance, M. \& Yadigaroglu 2009 Turbulent transport mechanisms in oscillating bubble plumes. J. Fluid Mech. 633, 191-231.

Socolofsky, S. A., AdAms, E. E. \& Sherwood, C. R. 2011 Formation dynamics of subsurface hydrocarbon intrusions following the deepwater horizon blowout. Geophys. Res. Lett. 38 (9), L09602.

Socolofsky, S. A. \& Bhaumik, T. 2008 Dissolution of direct ocean carbon sequestration plumes using an integral model approach. J. Hydraul. Engng ASCE 134 (11), 1570-1578.

Soga, C. \& Rehmann, C. 2004 Dissipation of turbulent kinetic energy near a bubble plume. J. Hydraul. Engng 130 (5), 441-449.

Sun, T.-Y. \& FAeth, G. M. 1986 Structure of turbulent bubbly jets. II. Phase property profiles. Intl J. Multiphase Flow 12 (1), 115-126.

TAN, C.-Y. \& HUANG, Y.-X. 2015 Dependence of refractive index on concentration and temperature in electrolyte solution, polar solution, nonpolar solution, and protein solution. J. Chem. Engng Data 60 (10), 2827-2833.

Wain, D. J. \& Rehmann, C. R. 2005 Eddy diffusivity near bubble plumes. Water Resour. Res. 41 (9), W09409.

Wang, B., Socolofsky, S. A., Breier, J. A. \& Seewald, J. S. 2016 Observations of bubbles in natural seep flares at MC 118 and GC 600 using in situ quantitative imaging. J. Geophys. Res. 121, 2203-2230.

WANG, H. \& LAW, A. W. K. 2002 Second-order integral model for a round turbulent buoyant jet. J. Fluid Mech. 459, 3.

Westerweel, J. \& SCARAno, F. 2005 Universal outlier detection for PIV data. Exp. Fluids 39 (6), 1096-1100.

Woods, A. W. 2010 Turbulent plumes in nature. Annu. Rev. Fluid Mech. 42, 391-412. 